\title{
Histone demethylase JMJD2A drives prostate tumorigenesis through transcription factor ETV1
}

\author{
Tae-Dong Kim, ${ }^{1}$ Fang Jin, ${ }^{2}$ Sook Shin,, ${ }^{1,3}$ Sangphil Oh, ${ }^{1}$ Stan A. Lightfoot, ${ }^{4}$ Joseph P. Grande, ${ }^{5}$ Aaron J. Johnson, ${ }^{2}$ \\ Jan M. van Deursen, ${ }^{6}$ Jonathan D. Wren, ${ }^{3,7}$ and Ralf Janknecht ${ }^{1,3}$

\begin{abstract}
'Department of Cell Biology, University of Oklahoma Health Sciences Center, Oklahoma City, Oklahoma, USA. ²Department of Immunology, Mayo Clinic, Rochester, Minnesota, USA. ${ }^{3}$ Stephenson Cancer Center, Oklahoma City, Oklahoma, USA. ${ }^{4}$ Department of Pathology, University of Oklahoma Health Sciences Center, Oklahoma City, Oklahoma, USA. ${ }^{5}$ Department of Laboratory Medicine and Pathology and ${ }^{6}$ Department of Biochemistry and Molecular Biology, Mayo Clinic, Rochester, Minnesota, USA. ${ }^{7}$ Arthritis and Clinical Immunology Research Program, Oklahoma Medical Research Foundation, Oklahoma City, Oklahoma, USA.
\end{abstract}

\begin{abstract}
Histone demethylase upregulation has been observed in human cancers, yet it is unknown whether this is a bystander event or a driver of tumorigenesis. We found that overexpression of lysine-specific demethylase 4A (KDM4A, also known as JMJD2A) was positively correlated with Gleason score and metastasis in human prostate tumors. Overexpression of JMJD2A resulted in the development of prostatic intraepithelial neoplasia in mice, demonstrating that JMJD2A can initiate prostate cancer development. Moreover, combined overexpression of JMJD2A and the ETS transcription factor ETV1, a JMJD2A-binding protein, resulted in prostate carcinoma formation in mice haplodeficient for the phosphatase and tensin homolog (Pten) tumor-suppressor gene. Additionally, JMJD2A cooperated with ETV1 to increase expression of yes associated protein 1 (YAP1), a Hippo pathway component that itself was associated with prostate tumor aggressiveness. ETV1 facilitated the recruitment of JMJD2A to the YAP1 promoter, leading to changes in histone lysine methylation in a human prostate cancer cell line. Further, YAP1 expression largely rescued the growth inhibitory effects of JMJD2A depletion in prostate cancer cells, indicating that YAP1 is a downstream effector of JMJD2A. Taken together, these data reveal a JMJD2A/ETV1/YAP1 axis that promotes prostate cancer initiation and that may be a suitable target for therapeutic inhibition.
\end{abstract}

\section{Introduction}

Prostate tumors are the most frequently diagnosed cancer in US men and a major health problem throughout the world. Apart from surgery and radiotherapy, androgen ablation is a standard treatment for advanced prostate cancer. However, patients with metastases generally relapse and die soon thereafter (1). The current lack of other effective therapies highlights the dire need for new drug targets to combat metastatic prostate cancer.

Deletion of tumor suppressors such as phosphatase and tensin homolog (PTEN), tumor protein p53 (TP53), or NK3 homeobox 1 (NKX3-1) is common in prostate cancer and linked to its etiology (2). On the other hand, various oncogenes are mutated or overexpressed in prostatic neoplasias, but arguably most prevalent are transcription factor Ets (ETS) chromosomal translocations that lead to the overexpression of DNA-binding ETS transcription factors (3-5). ETS genes most frequently translocated are v-ets avian erythroblastosis virus E26 oncogene homolog $(E R G)$ and ets variant 1 (ETV1). Compared with $E R G$, ETV1 upregulation correlates with more relapse after radical retropubic prostatectomy, is more enriched in metastases, and results in poorer disease-free survival in conjunction with PTEN loss $(6,7)$, suggesting that ETV1 translocations mark highly aggressive prostate tumors.

Conflict of interest: R. Janknecht is a scientific advisor for Chrysalis Therapeutics. Submitted: October 6, 2015; Accepted: November 13, 2015.

Reference information: / Clin Invest. 2016;126(2):706-720. doi:10.1172/JCI78132.
Aside from genetic defects, epigenetic changes underlie tumor development. Accordingly, drugs influencing the epigenetic state of a cell, such as histone deacetylase inhibitors, have proven to be valuable in the therapy of some cancers (8). Notably, changes of acetylation and methylation on specific histone residues were identified as predictors of prostate cancer recurrence $(9,10)$. This implies that modulating histone posttranslational modifications may be effective in restricting prostate tumor growth.

Histone lysine methylation was only recently recognized as an important posttranslational modification in cancer (11). However, histone demethylation and especially the corresponding demethylases have remained immensely understudied in prostate tumors. The vast majority of histone demethylases belong to the family of Jumonji C domain containing (JMJD) proteins (12). One demethylase subfamily consists of the 4 homologous JMJD2A-D proteins, also called lysine-specific demethylase 4A (KDM4A) (13). Here, we show how JMJD2A/KDM4A can exert its cellular functions through interaction with ETV1 and induction of the Hippo pathway component yes associated protein 1 (YAP1). In addition, we demonstrate for what we believe is the first time that overexpression of a histone demethylase (JMJD2A) may be an underlying cause of tumorigenesis, thereby highlighting JMJD2A as a valid anticancer drug target.

\section{Results}

JMJD2A interacts with ETV1. In our longstanding pursuit to mechanistically understand the action of the ETS transcription factor 

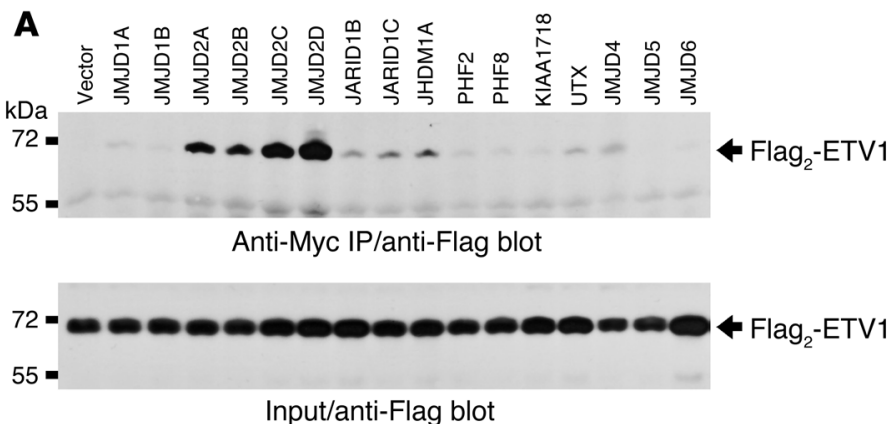

C
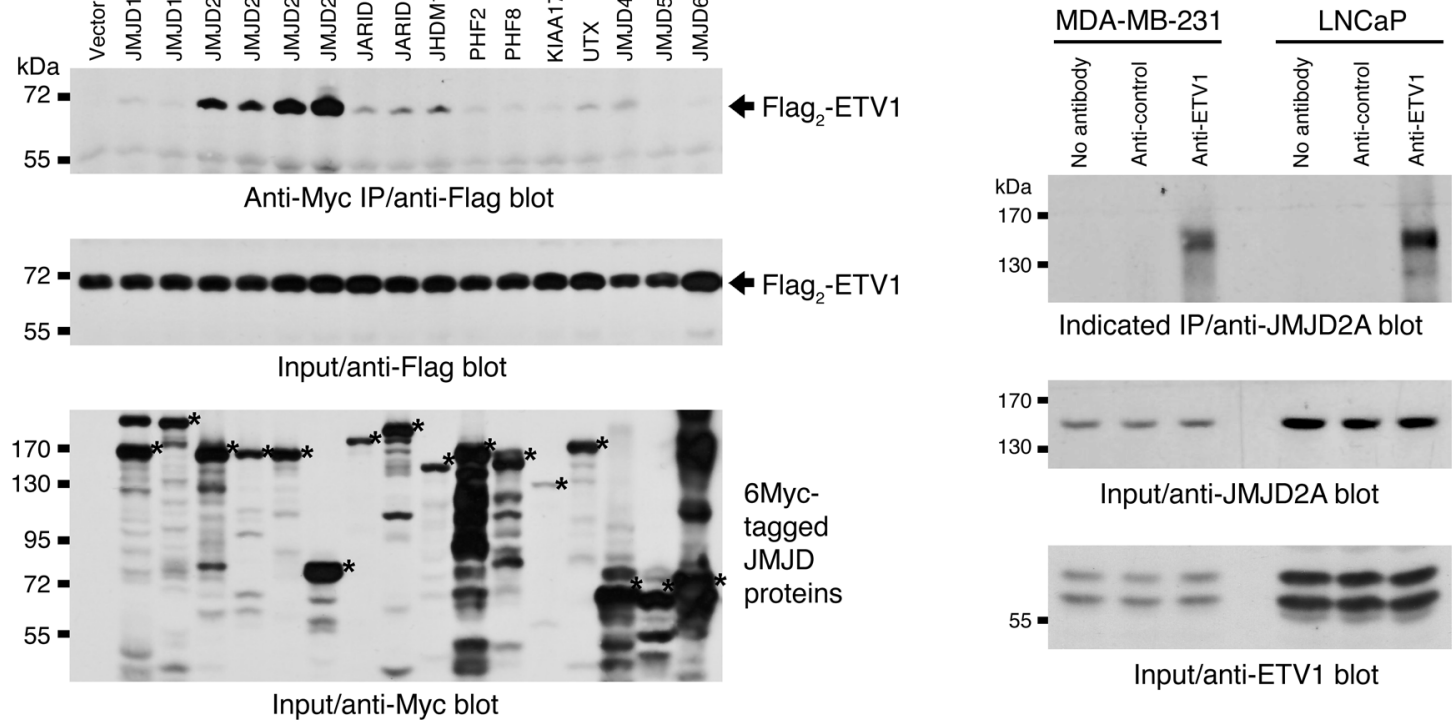

B 口vector

D
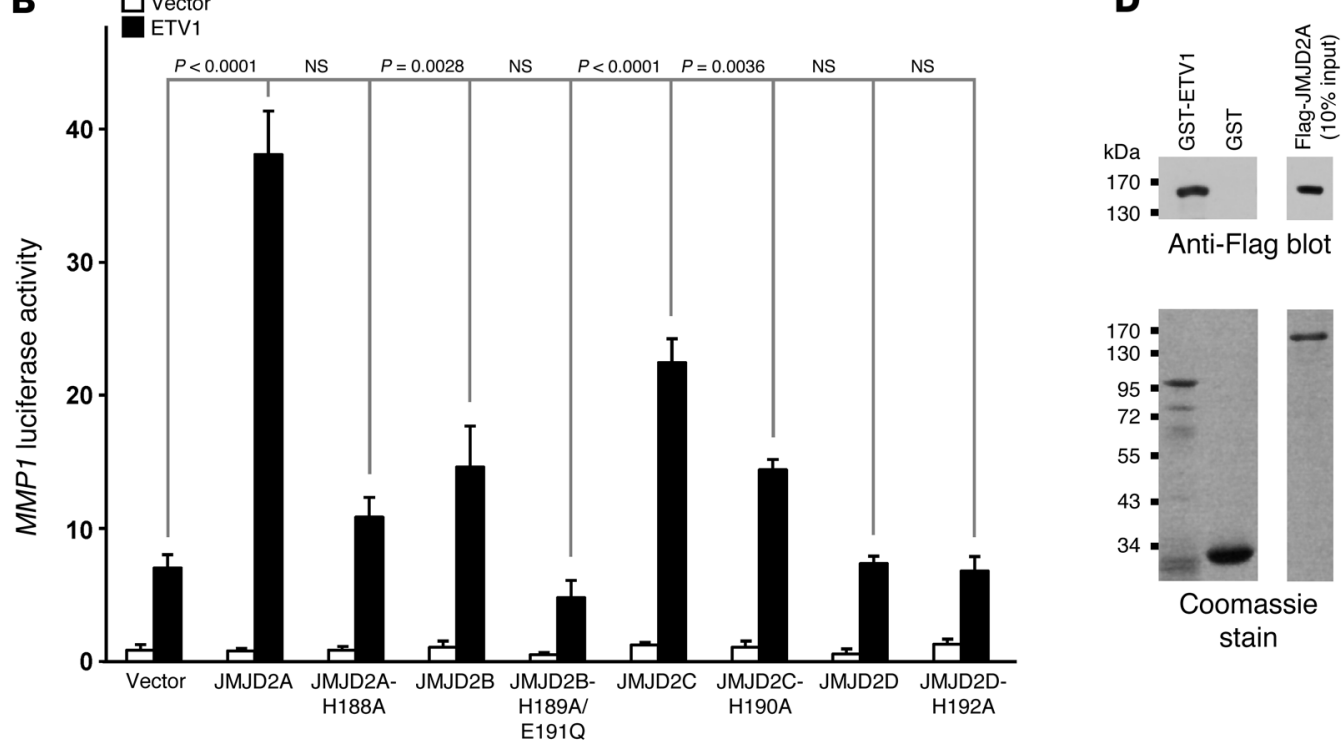

Figure 1. Interaction of JMJD2A with ETV1. (A) Coimmunoprecipitation assays of Flag 2 -ETV1 with 16 different 6Myc-tagged JMJD proteins (denoted by asterisks) in 293T cells. (B) Activation of an MMP1 luciferase reporter in BPH-1 cells. Luciferase activities shown are the average of 3 biological replicates with SD evaluated with 1-way ANOVA. (C) JMJD2A coprecipitates with ETV1 in MDA-MB-231 and LNCaP cells. (D) In vitro interaction of purified, Flag-tagged JMJD2A with GST-ETV1 (top). Coomassie-stained gels show the purity and relative amounts of utilized proteins (bottom).

ETV1, we tested whether it interacts with JMJD histone demethylases. Specifically, we coexpressed Flag-tagged ETV1 with 16 different Myc-tagged JMJD proteins representing all major JMJD subfamilies. The Myc-tagged JMJD proteins were immunoprecipitated with Myc Abs, and the resulting immunoprecipitates were probed with anti-flag Western blotting to determine which JMJD proteins interacted with ETV1 (Figure 1A). Notably, strong complex formation was only observable between ETV1 and the 4 JMJD2 proteins. Next, we analyzed whether JMJD2 proteins augment ETV1 in upregulating matrix metalloproteinase-1 (MMP1), a previously identified target gene of ETV1 (14). As we expected, ETV1 stimulated an MMP1 luciferase reporter gene in benign human $\mathrm{BPH}-1$ prostate cells (Figure 1B). Importantly, JMJD2A-C, but not JMJD2D, enhanced ETV1 activity, whereas all 4 JMJD2 proteins displayed negligible effects in the absence of ETV1. Further, JMJD2A was the most effective coactivator of ETV1, stimulating its activity by approximately 5.3-fold; please note that protein levels of JMJD2A-D were comparable (Supplemental Figure 1A; supplemental material available online with this article; doi:10.1172/ JCI78132DS1). We also tested a point mutant of JMJD2A, H188A, which is impaired in its catalytic activity $(15,16)$. In contrast to WT JMJD2A, this H188A mutant was much less able to cooperate with ETV1 (Figure 1B), yet still increased ETV1-dependent activity by approximately 1.5 -fold (although this was not statistically significant). Likewise, only JMJD2A, but not the H188A mutant, synergized with ETV1 to stimulate an MMP1 luciferase reporter 

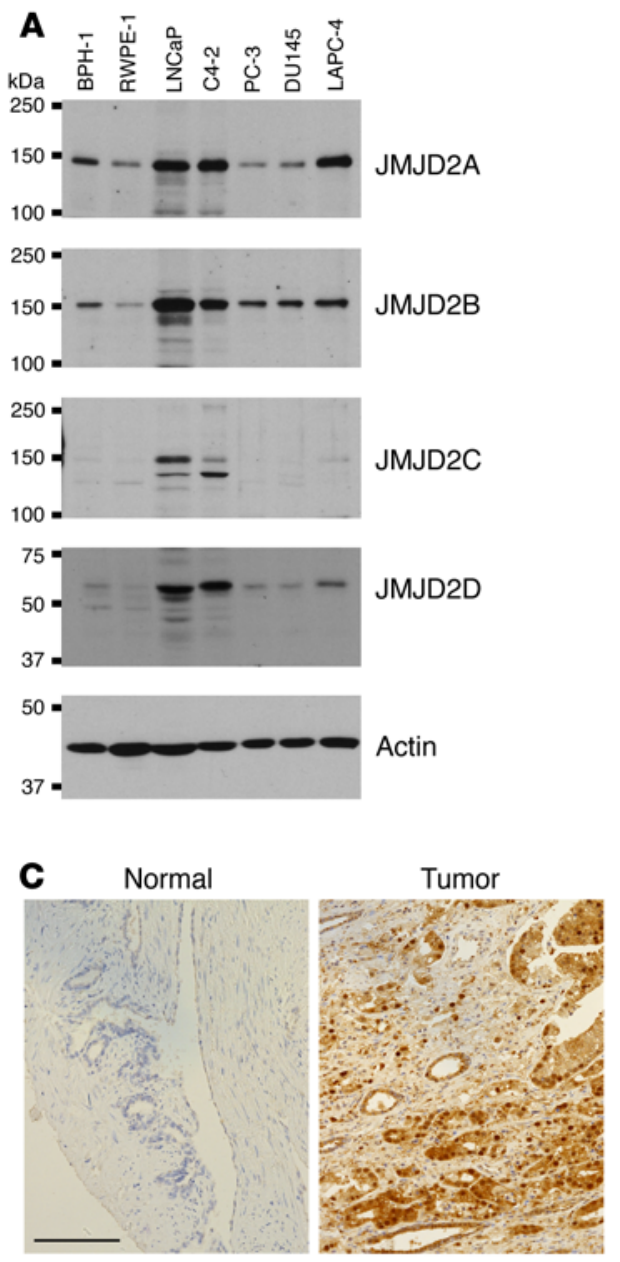
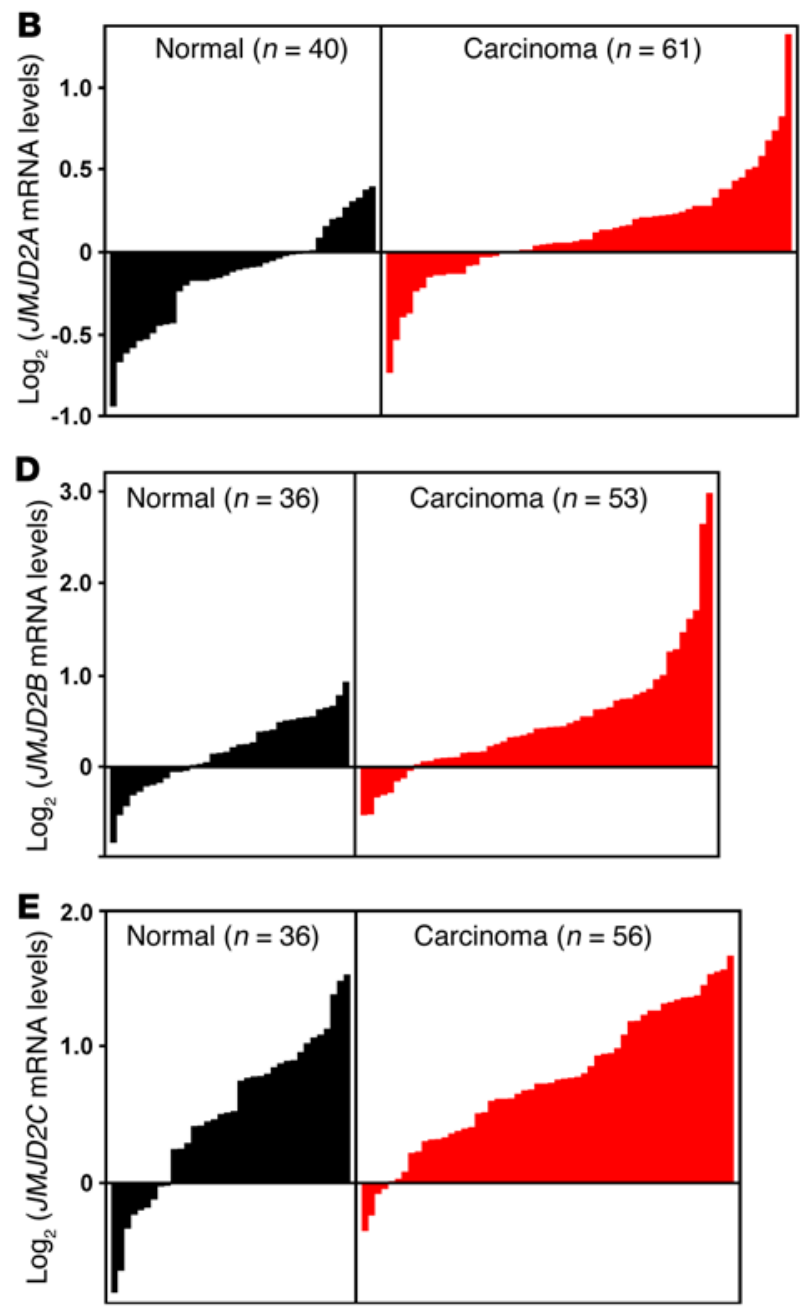

Figure 2. JMJD2 expression in the human prostate. (A) Western blots showing JMJD2 protein levels in untransformed (BPH-1, RWPE-1) and cancerous prostate cells (LNCaP, C4-2, PC-3, DU145, LAPC-4). (B) Different JMJD2A mRNA levels in 40 normal compared with 61 cancerous prostate tissues $\left(P=3.8 \times 10^{-5}\right.$; Student's $t$ test). Each bar represents 1 patient. (C) Example of immunohistochemical JMJD2A staining in matching normal and cancerous prostate tissue. Scale bar: $0.1 \mathrm{~mm}$. (D and E) Analogous to panel B for JMJD2B $(P=0.003)$ and $J M J D 2 C(P=0.021)$.

gene in African green monkey CV-1 kidney cells or an endogenous MMP1 gene transcription in human embryonic kidney $293 \mathrm{~T}$ cells (Supplemental Figure 1, B and C). Similarly, we observed that catalytically inactive JMJD2C-H190A, but not the corresponding JMJD2B-H189A/E191Q or JMJD2D-H192A mutants, stimulated ETV1 (Figure 1B). These data suggest that JMJD2A and JMJD2C stimulate ETV1 transcriptional activity predominantly in a manner dependent on their catalytic activity.

We then focused on the seemingly most potent transactivating JMJD2 protein and confirmed that endogenous JMJD2A also interacted with endogenous ETV1 in MDA-MB-231 breast and LNCaP prostate cancer cells (Figure 1C). Further, we purified GST-tagged ETV1 from bacteria and Flag-tagged JMJD2A from baculovirus and observed that Flag-JMJD2A was retained on glutathione beads loaded with GST-ETV1, but not with the GST moiety (Figure 1D). This shows that JMJD2A and ETV1 can directly bind to each other. Moreover, we found that the C-terminal amino acids 333-477, which encompass ETV1's DNA-binding and C-terminal activation domains, mediated the interaction with JMJD2A
(Supplemental Figure 1D). Conversely, the middle portion of JMJD2A (amino acids 490-750) was sufficient for binding ETV1 (Supplemental Figure 1E). Together, our data strongly suggest that JMJD2A is a bona fide coactivator of ETV1.

JMJD2A overexpression in prostate tumors. ETV1 is most prominently implicated in tumor formation within the prostate. Therefore, we explored whether JMJD2A would be expressed in this organ. First, utilizing specific JMJD2A Abs (see Supplemental Figure 2), we analyzed JMJD2A protein levels in several human prostate cell lines and observed elevated expression in some tumor-derived compared with 2 untransformed prostate cell lines (Figure 2A). Second, we evaluated levels of JMJD2A mRNA. Consistent with a previous report (17), Oncomine analysis of published microarray data (18) revealed that JMJD2A mRNA levels were significantly enhanced in prostate carcinomas relative to normal prostates (Figure 2B); this was corroborated with various other microarray data sets (Supplemental Figure 3, A and B) and was not due to JMJD2A gene copy number changes (Supplemental Figure $3 C$ ). Notably, JMJD2A expression was high in most pros- 

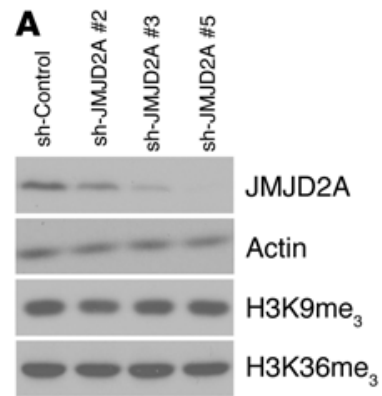

C

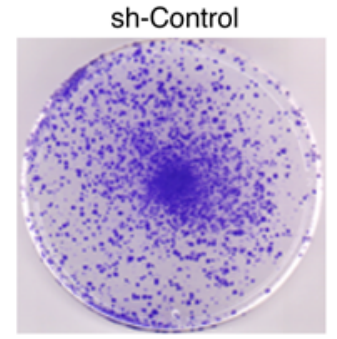

D

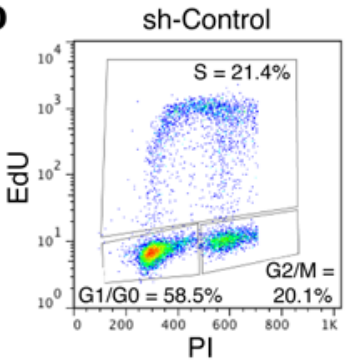

B

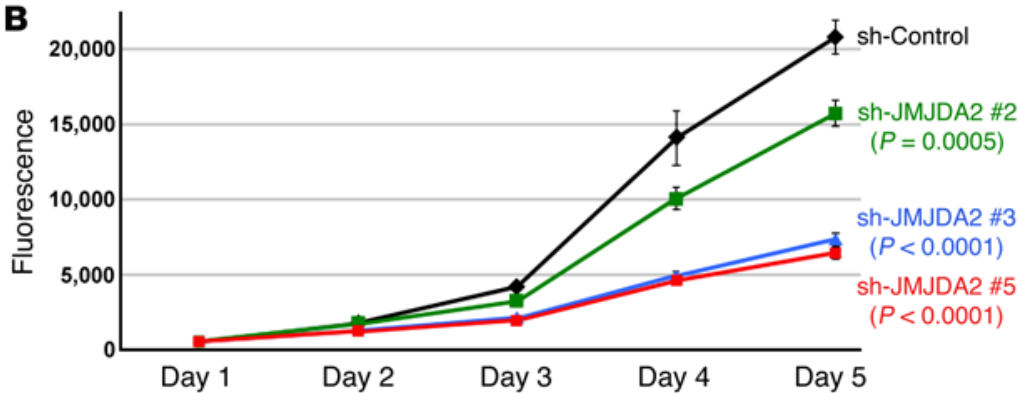

Day 1
sh-JMJD2A \#2

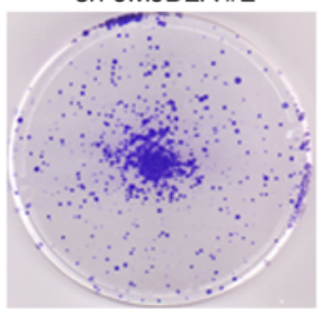

sh-JMJD2A \#2

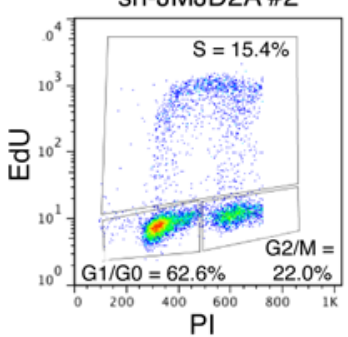

sh-JMJD2A \#3

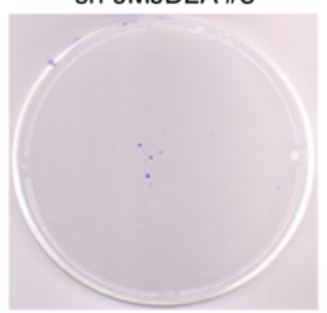

sh-JMJD2A \#5

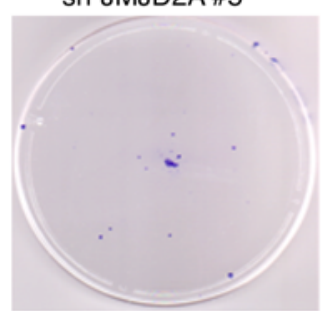

sh-JMJD2A \#3
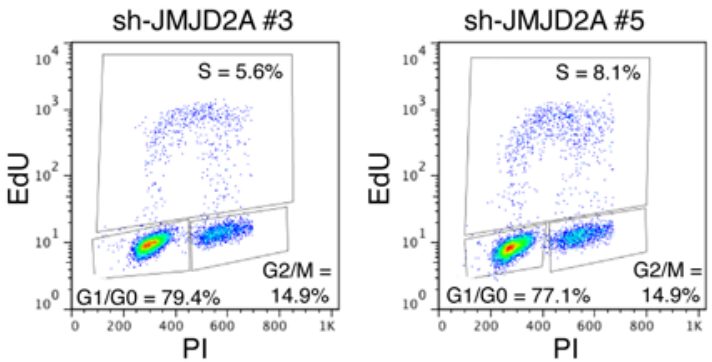

Figure 3. Role of JMJD2A in LNCaP prostate cancer cells. (A) Downregulation of JMJD2A in LNCaP cells with 3 different shRNAs. Western blots show levels of indicated proteins. (B) Corresponding cell growth assays. Shown are averages ( $n=3$ per group) with SD. Statistical significance of growth differences (compared with sh-Control) at day 5 was evaluated with 1-way ANOVA. Representative of at least 3 independent experiments. (C) Representative (out of 3 ) clonogenic assays. (D) Cell-cycle distribution (representative out of 2 independent experiments) in the presence of JMJD2A shRNA.

tate tumors with ETV1 translocations (Supplemental Figure 4). Less pronounced, JMJD2A expression was also increased in the benign precursors of prostate carcinoma, hyperplasia and prostatic intraepithelial neoplasia (PIN) (Supplemental Figure 5A). Third, we stained human prostate tissue microarrays with JMJD2A Abs. Strong nuclear staining of JMJD2A was observable in many prostate tumors, but rarely in matching normal tissue (Figure $2 \mathrm{C}$ and Supplemental Figure 5B). Overall, high nuclear JMJD2A protein expression was found in $81 \%$ of tumors versus $3 \%$ of normal prostate tissues. Moreover, nuclear JMJD2A protein staining positively correlated with the Gleason score (Supplemental Figure 6A) as did JMJD2A mRNA levels (Supplemental Figure 6B). Also, compared with the primary prostate tumor site, metastases displayed higher JMJD2A expression (Supplemental Figure 6C). All this suggests that JMJD2A expression increases during the progression of prostate cancer development and might therefore be a marker for aggressive prostate tumors.

Similar to JMJD2A, we found overexpression of the other JMJD2 proteins in some of the prostate cancer cell lines, yet this in itself cannot be regarded as significant due to the low number of cell lines tested (Figure 2A). Further, JMJD2B and JMJD2C mRNA levels were also enhanced in prostate tumors in multiple data sets (Figure 2, D and E, and Supplemental Figure 7, A and B).
However, we found only one microarray experiment with Oncomine that showed a significant change of JMJD2D mRNA levels in prostate carcinoma, and unlike with JMJD2A-C, JMJD2D levels were decreased in prostate tumors compared with those in normal prostate tissue (Supplemental Figure 7C). These data suggest that JMJD2B and JMJD2C, but not JMJD2D, may also be overexpressed in prostate cancer.

JMJD2A stimulates prostate cell proliferation. As a first step to test whether JMJD2A might be causally implicated in prostate tumor development, we examined JMJD2A's impact on cell growth. We focused on LNCaP prostate cancer cells, since they are characterized by ETV1 overexpression $(19,20)$ and express JMJD2A at a high level (see Figure 2A). Three different JMJD2A shRNAs were expressed in LNCaP cells, which reduced JMJD2A protein levels with different efficiencies (Figure $3 \mathrm{~A}$ ). Although JMJD2A is capable of demethylating $\mathrm{H} 3 \mathrm{~K}_{9} \mathrm{me}_{3}$ or $\mathrm{H} 3 \mathrm{~K} 36 \mathrm{me}_{3}(15,16)$, these epigenetic marks were not globally affected by JMJD2A depletion (Figure 3A). Yet JMJD2A downregulation resulted in reduced cell growth (Figure 3B) and clonogenic capacity (Figure 3C). Consistent with being least able to reduce JMJD2A levels, shRNA no. 2 was less effective than the other 2 JMJD2A shRNAs in these assays. These progrowth characteristics of JMJD2A were also observed in other prostate cancer cell lines (C4-2, PC-3, DU145, LAPC-4) and 
A

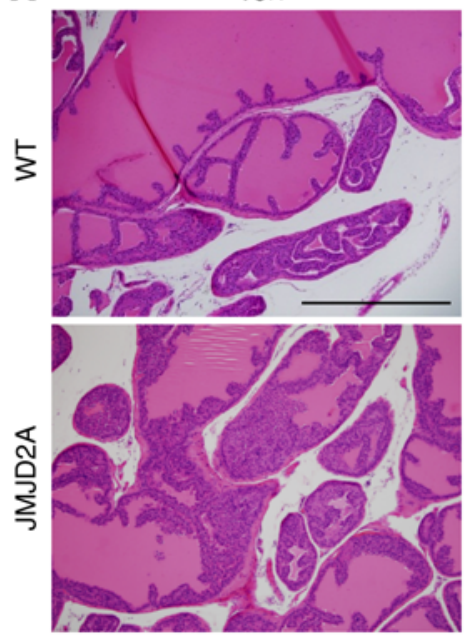

$40 x$

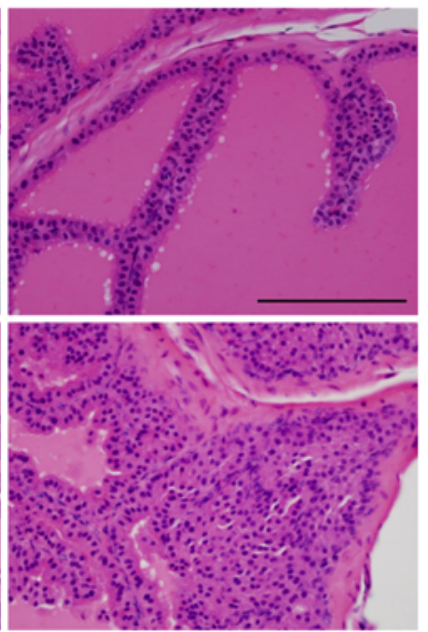

B

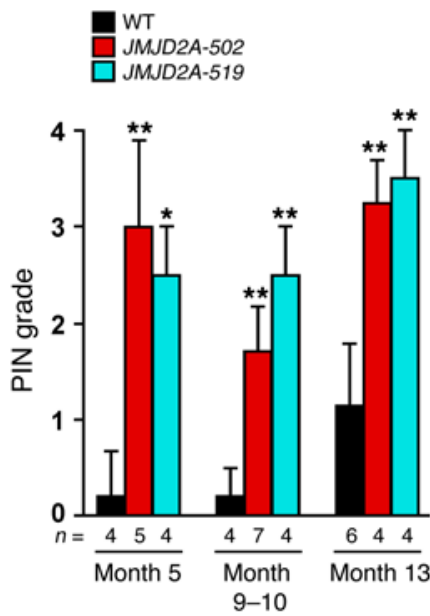

C

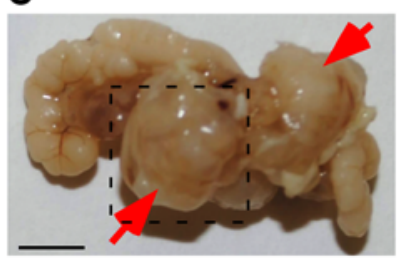

D

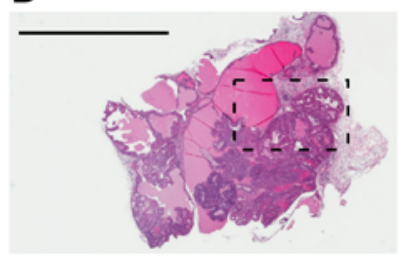

E

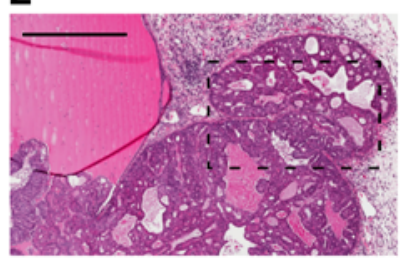

$\mathbf{F}$

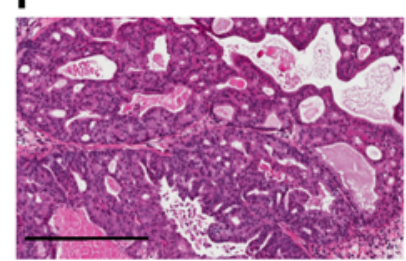

Figure 4. Prostate phenotypes of transgenic JMJD2A mice. (A) H\&E staining of a sectioned prostate (anterior lobe) from a WT or JMJD2A transgenic mouse. Scale bars: $0.5 \mathrm{~mm}$ (left); $0.125 \mathrm{~mm}$ (right). (B) Summary of PIN formation in WT mice and 2 independent transgenic JMJD2A lines (502 and 519 ). Shown are PIN grade averages with SD and number of animals at 3 different ages. ${ }^{*} P<0.005$; ${ }^{*} P<0.001,1$-way ANOVA. (C) Prostate with bladder and seminal vesicles from a JMJD2A/ETV1/Pten ${ }^{+-}$mouse; arrows point to tumor masses. Scale bar: $5 \mathrm{~mm}$. (D-F) Progressively higher magnifications of H\&Estained sections of the lower tumor mass shown in C. Scale bars: $3 \mathrm{~mm}$ (D); $0.6 \mathrm{~mm}(\mathbf{E}) ; 0.3 \mathrm{~mm}$ (F).

normal BPH-1 and RWPE-1 prostate cells (Supplemental Figure 8) as well as in LNCaP cells deprived of or stimulated with an androgen (Supplemental Figure 9, A and B). Similarly to what occurred with JMJD2A, downregulation of JMJD2B or JMJD2C also resulted in reduced LNCaP cell growth (Supplemental Figure 9, C and D).

Cell-cycle profiling revealed that JMJD2A downregulation strongly reduced the number of LNCaP cells in the S phase, whereas the $G_{2} / M$ proportion was only moderately affected (Figure $3 D$ ). There were no detectable signs of PARP cleavage nor significant changes in sub- $G_{1} / G_{0}$ DNA content cells (Supplemental Figure 10), indicating that the observed growth defects were due to deficient cell proliferation and not increased apoptosis. In conclusion, consistent with a potential role in driving tumorigenesis, JMJD2A is a positive regulator of proliferation in prostate cells.

Interestingly, overexpression of JMJD2A in LNCaP cells significantly enhanced their growth (Supplemental Figure 11, A and B), whereas the H188A catalytically inactive mutant suppressed LNCaP cell growth, indicating that it may act as a dominant-negative protein. In contrast, overexpression of neither WT JMJD2A nor its H188A mutant had a growth effect in normal BPH-1 cells (Supplemental Figure 11, C and D). This suggests that catalytic activity of JMJD2A may be needed for maximal growth of cancerous but not normal prostate cells.

Analysis of JMJD2A transgenic mice. To assess the role of JMJD2A overexpression in vivo, we created transgenic mice that expressed JMJD2A under the control of the prostate-specific rat probasin promoter. We obtained 4 founder mice, which all developed PIN. Two founder mice had offspring with a JMJD2A transgene (Supplemental Table 1); JMJD2A transgene expression was negligible in organs other than the prostate, with the exception of seminal vesicles (Supplemental Figure 12A), which are androgen receptor-positive and therefore also display slight induction of the probasin promoter (21). Cohorts of these 2 independent JMJD2A transgenic lines were established, which displayed enhanced JMJD2A staining, particularly within PIN regions (Supplemental Figure 12B). Likewise, the cell proliferation marker Ki67 was expressed in a more pronounced manner in neoplastic regions of prostates from JMJD2A transgenic mice (Supplemental Figure 12C). Also, staining for smooth muscle actin, which is normally found in the continuous fibromuscular layer surrounding the ducts but may become discontinuous upon PIN formation, often displayed discontinuities around diseased areas in prostates from JMJD2A mice (Supplemental Figure 12D). More systematically, PIN formation was classified on a scale of 1 to 4 as defined for genetically engineered mice (22). Already at 5 months of age, many of the JMJD2A transgenic mice developed high-grade PIN (grades 3 and 4), whereas only 1 syngeneic age-matched WT control mouse presented with grade 1 PIN and 3 further control mice had no PIN at all (Figure 4, A and B, and Supplemental Table 1). At 13 months of age, all transgenic mice presented with high-grade PIN; such lesions were not detected in age-matched control mice, although two-thirds of these mice exhibited low-grade PIN. These 
Table 1. Tumor formation in compound mice

\begin{tabular}{|c|c|c|c|c|}
\hline Genotype & No. of mice & $\begin{array}{l}\text { Macroscopic and/or } \\
\text { microscopic carcinoma }\end{array}$ & Z score & $P$ value $^{A}$ \\
\hline JMID2A/ETV1/Pten ${ }^{+-}$ & 13 & 8 & & \\
\hline JMJD2A/Pten ${ }^{+/-}$ & 7 & 0 & 2.68 & 0.007 \\
\hline ETV1/Pten ${ }^{+/-}$ & 7 & 1 & 2.03 & 0.042 \\
\hline JMJD2A/ETV1 & 6 & 0 & 2.53 & 0.011 \\
\hline
\end{tabular}

results demonstrate that $J M J D 2 A$ initiates neoplastic growth when overexpressed in the prostate.

However, we did not observe any prostate carcinomas in our JMJD2A transgenic mice, which is similar to what has been observed in ETV1 transgenic mice that also only develop PIN (6, 20). This suggests that JMJD2A or ETV1 overexpression primes prostate cells for the transformation from the PIN to the carcinoma stage upon acquisition of further mutations. PTEN mutations are frequently observed at the beginning and even more so during malignant progression of human prostate cancer, and Pten knockout can synergize with other genetic events in inducing murine carcinomas (2). Two models are commonly used: heterozygous $\mathrm{Pten}^{+/-}$mice, which in contrast to Pten ${ }^{-/}$mice are viable and develop PIN, and Pten flox/flox mice, in which prostate-specific inactivation of both Pten alleles induces invasive prostate carcinomas (23, 24). We elected to utilize the heterozygous $\mathrm{Pten}^{+/-}$mouse model, since it may more faithfully reflect prostate cancer progression in humans that does not entail simultaneous inactivation of both PTEN alleles. In JMJD2A/Pten ${ }^{+/-}$compound mice that we generated, PIN formation, but no carcinomas, was observable (Table 1 and Supplemental Figure 13). Similarly, ETV1/Pten ${ }^{+/}$mice reportedly solely develop PIN (7), and our data also show predominantly PIN formation with the exception of 1 ETV1/Pten ${ }^{+-}$mouse that developed a minor, very focal carcinoma (Table 1 ).

We then reasoned that rather than JMJD2A alone, the JMJD2AETV1 complex may cooperate with Pten haploinsufficiency to induce carcinomas. And indeed, while combined overexpression of JMJD2A and ETV1 did not induce carcinomas, 8 out of 13 triplemutant JMJD2A/ETV1/Pten ${ }^{+/-}$mice displayed prostate carcinoma formation; in 5 cases, tumor masses were even macroscopically visible on the triple-mutant prostates (Table 1, Figure 4, C-F, and Supplemental Figure 13). Together, these data indicate that the JMJD2AETV1 complex possesses oncogenic activity in the prostate.

Identification of JMJD2A target genes. To decipher how the JMJD2A-ETV1 complex exerts its oncogenic potential, we performed mRNA microarray experiments with $\mathrm{LNCaP}$ prostate cancer cells expressing JMJD2A or ETV1 shRNAs. Compared with control shRNA, 256 genes were at least 1.4-fold downregulated by each of the 2 JMJD2A shRNAs used (Figure 5A and Supplemental Table 2). Similarly, 2 different ETV1 shRNAs led to at least a 1.4fold reduced expression of 209 genes (Figure 5A and Supplemental Table 3). Of those, 97 were also downregulated with JMJD2A shRNAs, suggesting that these genes are jointly stimulated by JMJD2A and ETV1. On the other hand, we found that 212 or 196 genes were upregulated in the presence of JMJD2A or ETV1
shRNA, respectively; of these genes, 75 appeared to be coregulated by JMJD2A and ETV1. The number of genes jointly up- or downregulated with JMJD2A and ETV1 shRNAs was statistically significant $\left(P<0.0001, \chi^{2}\right.$ goodness-of-fit test), indicating that joint regulation of gene transcription by JMJD2A and ETV1 is common in LNCaP prostate cancer cells.

Ingenuity systems analysis of our microarray data indicated 3 upstream regulators whose target molecules were affected upon JMJD2A/ETV1 downregulation: VEGF, HGF, and TP53 (Supplemental Figure 14A). VEGF and $\mathrm{HGF}$ are both angiogenic growth factors and targets in clinical trials for prostate cancer (25), whereas TP53 is one of the most prominent tumor-suppressor molecules (26). Our data suggest that JMJD2A/ETV1 overexpression would result in activation of VEGF/HGF-mediated pathways, whereas the tumorsuppressive function of TP53 would be diminished, all of which could contribute to prostate tumorigenesis.

Among the most responsive common JMJD2A/ETV1 target genes (Supplemental Figure 14B and Supplemental Table 4), we selected 2 for further study, YAP1 and prostate transmembrane protein androgen induced 1 (PMEPA1). This selection was based on the facts that both genes are part of a network of JMJD2A/ETV1-regulated genes (Supplemental Figures 15 and 16) and that bioinformatical expression analyses (see below) or literature review predicted prostate cancer involvement. YAP1 is a transcriptional cofactor and downstream effector in the Hippo pathway that regulates organ growth and whose dysregulation is implicated in cancer $(27,28)$. PMEPA1 expression is regulated by the androgen receptor, and its overexpression can inhibit growth of prostate cancer cells $(29,30)$. We confirmed that downregulation of either ETV1 or JMJD2A reduced YAP1 mRNA and protein levels, whereas the opposite occurred for PMEPA1 (Figure 5, B and C, and Supplemental Figure 17). Furthermore, ChIP assays indicated that both ETV1 and JMJD2A bound to the YAP1 and PMEPA1 gene promoters (Figure 5D). As a control, we demonstrated that neither ETV1 nor JMJD2A bound to a YAP1 intron 3 region and only ETV1 bound to a region that is approximately $2.5 \mathrm{~kb}$ upstream of the YAP1 transcription start site, indicating that ETV1 and JMJD2A do not always bind to the same chromatin sites. Likewise, JMJD2A failed to bind to the promoter of the MMP7 matrix metalloproteinase (Figure 5D), a known ETV1 target gene (31), and accordingly, only ETV1, but not JMJD2A, shRNAs affected MMP7 mRNA levels (Figure 5B and Supplemental Figure 17), further validating our microarray screen. Together, these data suggest that the YAP1 and PMEPA1 promoters are directly regulated by a complex of ETV1 and JMJD2A.

We searched for additional evidence within public data sets to clarify whether the gene expression correlations identified above are general or might be specific to our experimental conditions by analyzing 3,949 human 2-color microarray data sets that represent a variety of normal and diseased tissues (32). Strong correlations were found between YAP1 and ETV1 as well as between YAP1 and $J M J D 2 A$ expression (Figure 5, $\mathrm{E}$ and $\mathrm{F}$ ). This underscores that regulation of the YAP1 promoter by JMJD2A/ETV1 is widespread and of global importance. Further, data from the Cancer Genome Atlas (http://cancergenome.nih.gov/) indicated that YAP1 and JMJD2A 
A sh- sh-

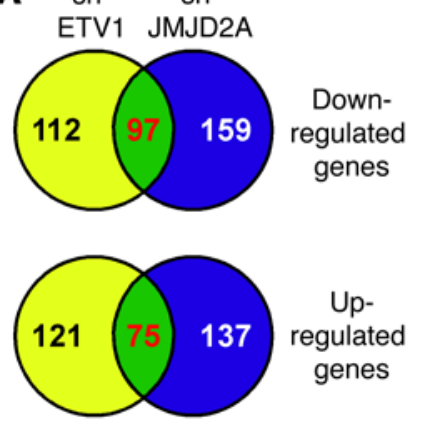

D

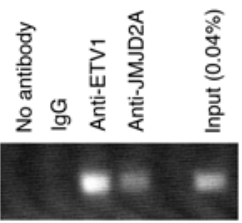
YAP1

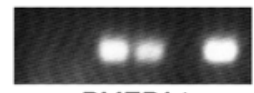

PMEPA1

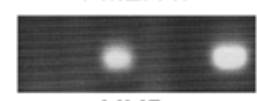

MMP7

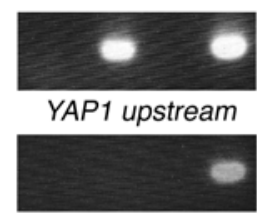

YAP1 intron 3
B

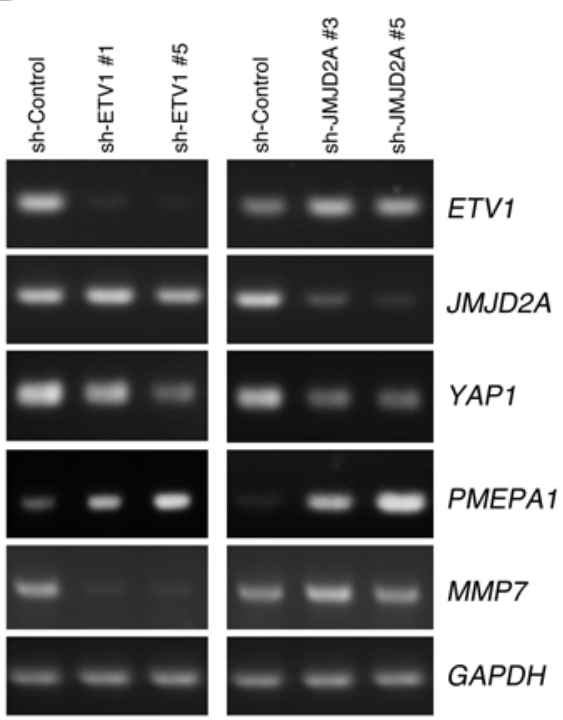

C

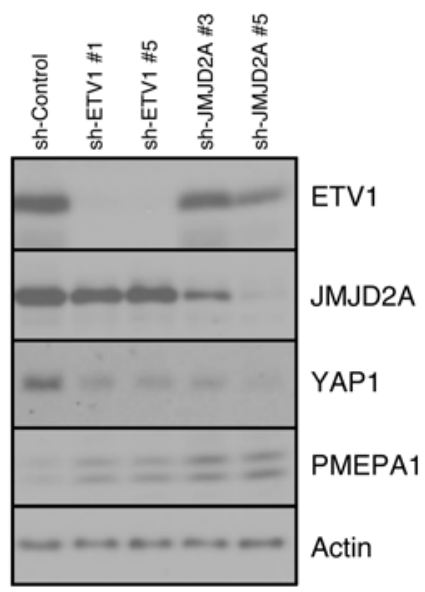

E

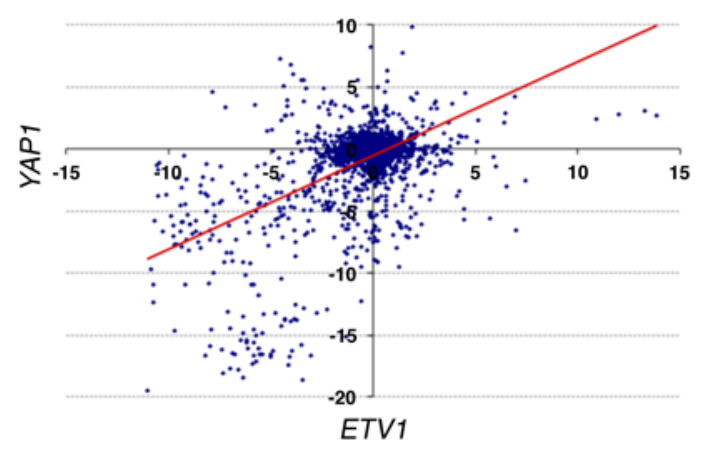

$\mathbf{F}$

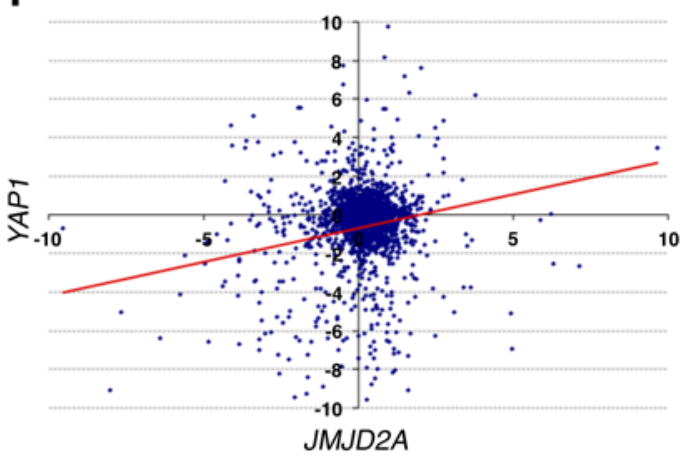

Figure 5. Identification of JMJD2A/ETV1 target genes. (A) Venn diagrams of genes down- or upregulated upon treatment of LNCaP cells with ETV1 or JMJD2A shRNAs. (B) Validation of target genes by RT-PCR in LNCaP cells. (C) Corresponding analysis of protein levels. (D) ChIP assay with ETV1 and JMJD2A Abs at indicated gene promoters in LNCaP cells. YAP1 upstream is approximately $2.5 \mathrm{~kb}$ upstream, and YAP1 intron 3 is approximately $72 \mathrm{~kb}$ downstream of the YAP1 transcription start site. (E) Correlation between fold changes in YAP1 and ETV1 mRNA levels across 3,949 microarrays. Pearson correlation: $R=0.52 ; P=4.64 \times 10^{-177}$. (F) Correlation between fold changes in YAP1 and JMJD2A mRNA levels across 3,949 microarrays. Pearson correlation: $R=0.12 ; P=2.29 \times 10^{-10}$.

mRNA levels are significantly correlated to each other in human prostate adenocarcinomas (Supplemental Figure 18). In contrast, there was no significant correlation between PMEPA1 and either JMJD2A or ETV1 in the 3,949 human 2-color microarray data sets, suggesting that PMEPA1 regulation by JMJD2A/ETV1 may be limited to the prostate, possibly because PMEPA1 is an androgendependent gene (29). Since we did not find any impact of PMEPA1 downregulation or overexpression on LNCaP cell growth (Supplemental Figure 19) in contrast with YAP1 (see below), we focused in the following on the analysis of YAP1 in prostate cancer cells.

Analysis of the YAP1 promoter. To corroborate that YAP1 is a direct target of ETV1 and JMJD2A, we fused the YAP1 promoter to a luciferase reporter gene and measured how ETV1 and JMJD2A would affect its activity. ETV1 or JMJD2A overexpression alone led to a robust activation of the YAP1 promoter in LNCaP cells, and the combination of ETV1 and JMJD2A was strongly collaborative (Figure 6A). In contrast, catalytically inactive JMJD2A-H188A stimulated the YAP1 promoter by much less. This indicates that JMJD2A promotes YAP1 transcription predominantly, but not absolutely, through its catalytic activity. JMJD2B and JMJD2C, which were expressed at levels comparable to those of JMJD2A (Supplemental Figure 20A), were much less efficient in coactivating ETV1, and their catalytic mutants were not at all active (Figure 6A). Further, JMJD2D had no significant impact on ETV1-mediated YAP1 promoter activity. This is similar to what we noted at the MMP1 promoter (see Figure 1B), further corroborating that among the JMJD2 proteins, JMJD2A appears to be the most potent coactivator of ETV1.

To prove that ETV1 can directly bind to the YAP1 promoter, we performed electrophoretic mobility shift assays with ${ }^{32} \mathrm{P}$-labeled oligonucleotides spanning potential ETV1-binding sites within the YAP1 promoter. While ETV1 bound to E74, a previously characterized ETV1-binding site (33), it did not bind to any of the 8 potential ETS sites present in the YAP1 promoter (Figure 6B and Supplemental Figure 20B). However, when we added an Ab that relieves an intramolecular inhibition of DNA binding (5), we found strong binding of ETV1 to 2 oligonucleotides spanning either the 

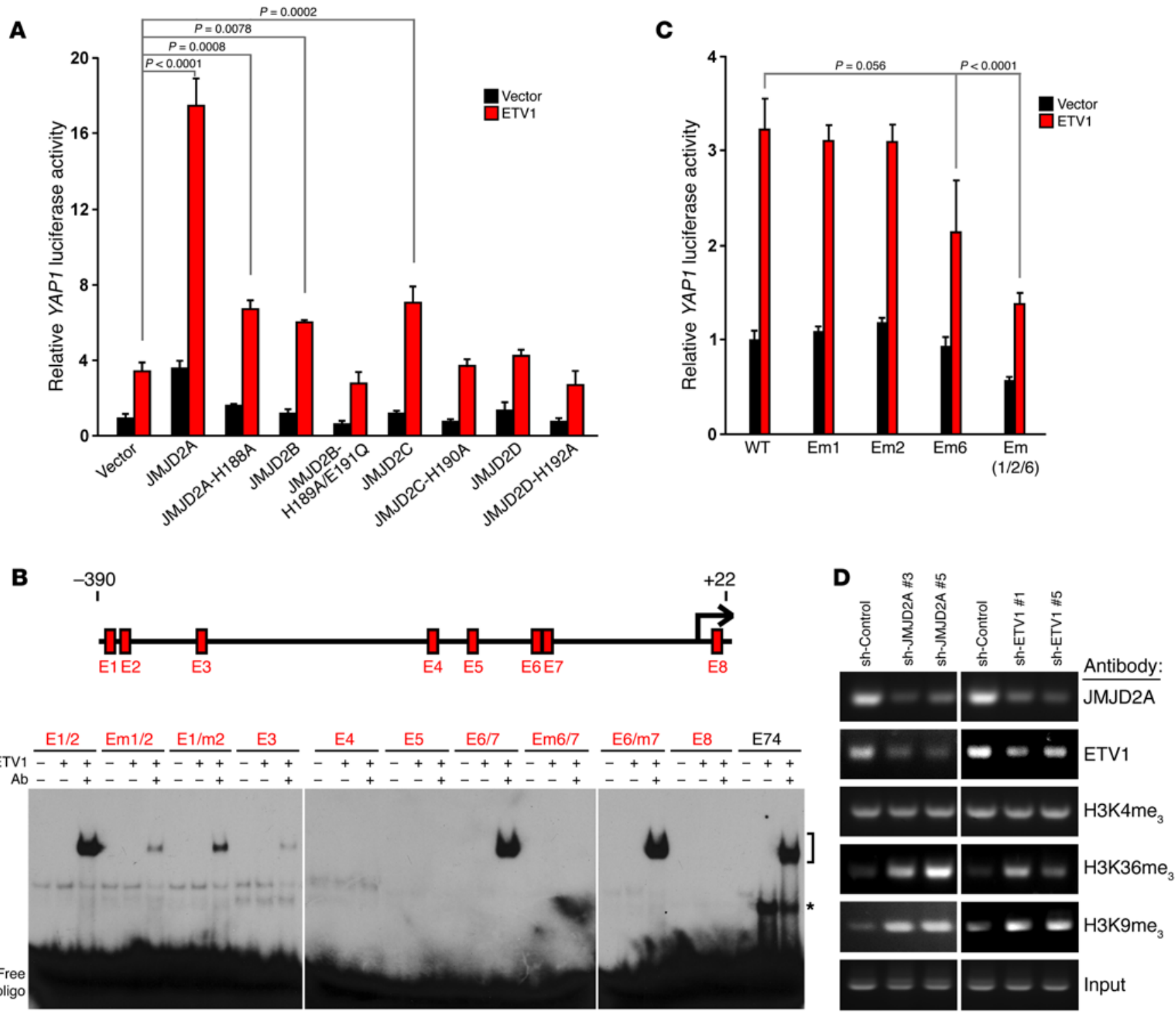

Figure 6. Characterization of the YAP1 promoter. (A) Activation of a YAP1 promoter (-390/+22) luciferase reporter by ETV1 and JMJD2 protein (or respective catalytic mutant) in LNCaP cells. Shown are averages ( $n=3$ biological replicates per group) with SD and 1-way ANOVA probabilities. (B) Electrophoretic mobility shift assays with ${ }^{32} \mathrm{P}$-labeled oligonucleotides corresponding to the 8 ETS sites (E1 to E8) within the YAP1 promoter or the E74 binding site. m, mutated ETS site; asterisk denotes an ETV1:DNA complex, whereas the bracket marks the supershift of ETV1:DNA complexes caused by an ETV1 Ab. (C) Impact of mutating E1, E2, and/or E6 on YAP1 promoter luciferase activity in LNCaP cells. Shown are averages ( $n=3$ biological replicates per group) with SD. Significance was determined with 1-way ANOVA. (D) YAP1 promoter ChIP assays with indicated Abs on LNCaP cells treated with JMJD2A or ETV1 shRNA. Amplified DNA fragments covered the promoter from -440 to -12 .

juxtaposed ETS site 1 (E1) and E2 or E6 and E7; this is reminiscent of induced in vitro DNA-binding of ETV1 to other target gene promoters, such as $M M P 7$ and $S M A D 7$, in the presence of this ETV1 $\mathrm{Ab}(31,34)$. Mutation of ETS site $1(\mathrm{Em} 1 / 2)$ or $2(\mathrm{E} 1 / \mathrm{m} 2)$ in the $\mathrm{E} 1 / 2$ oligonucleotide reduced ETV1 binding, as did mutation of E6, but not of $\mathrm{E} 7$, in the E6/7 oligonucleotide (Figure 6B). These results implicate E1, E2, and E6 as possibly responsible for recruiting ETV1 to the YAP1 promoter.

We then mutated E1, E2, and E6 and observed that only mutation of E6 reduced ETV1-stimulated YAP1 promoter activity (Figure $6 \mathrm{C}$ ), suggesting that E6 is the most relevant ETV1-binding site within the YAP1 promoter. However, when E1 and E2 were mutat- ed in addition to $\mathrm{E} 6$, there was a trend toward further reduction of YAP1 luciferase activity (Figure 6C), implying that E1 and E2 may also contribute to YAP1 promoter activity. Regardless, our DNAbinding experiments combined with the luciferase assays establish that ETV1 can directly regulate the YAP1 promoter.

Finally, we assessed how JMJD2A, which is a $\mathrm{H} 3 \mathrm{~K}_{9} \mathrm{me}_{3}$ and H3K36 $\mathrm{me}_{3}$ demethylase $(15,16)$, may affect histone methylation at the YAP1 promoter. Downregulation of JMJD2A led expectedly to less JMJD2A recruitment in vivo (Figure 6D and Supplemental Figure 21). Interestingly, binding of ETV1 to the YAP1 promoter was also reduced, suggesting that JMJD2A facilitates DNA binding of ETV1 in vivo. Furthermore, JMJD2A shRNAs caused an 
A

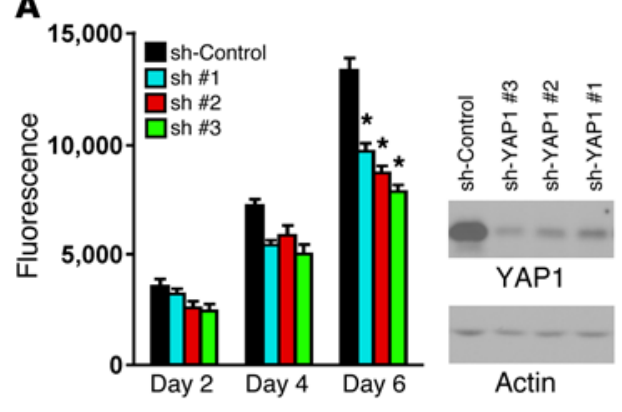

B

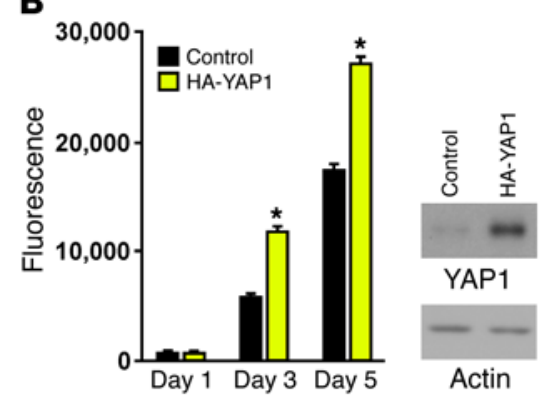

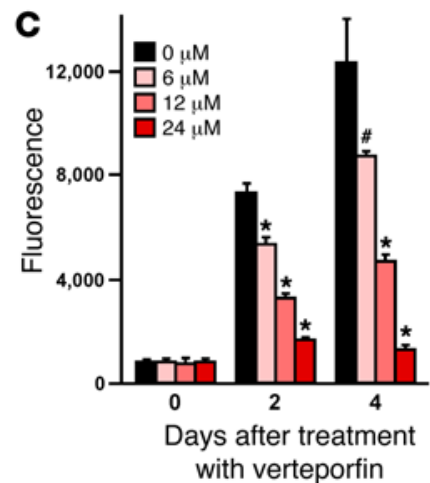
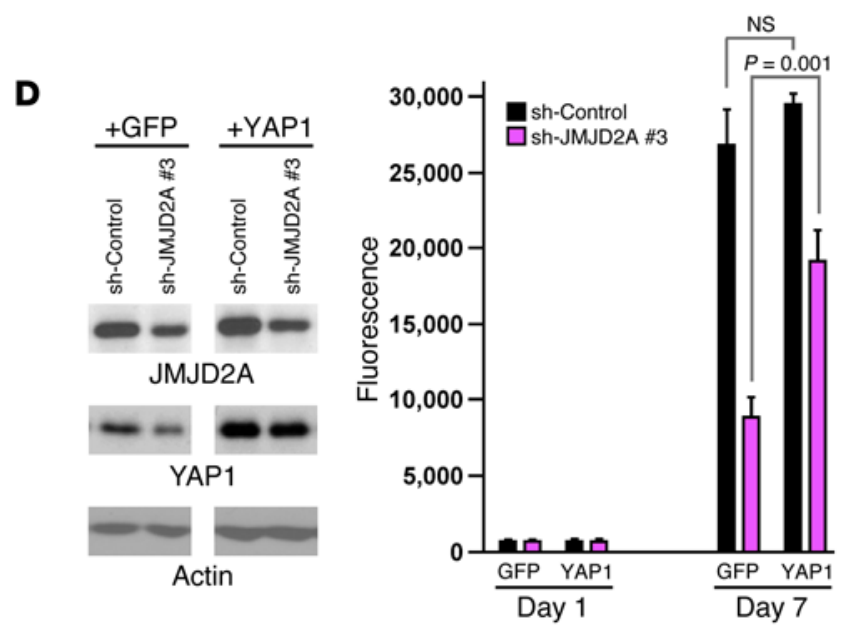

E

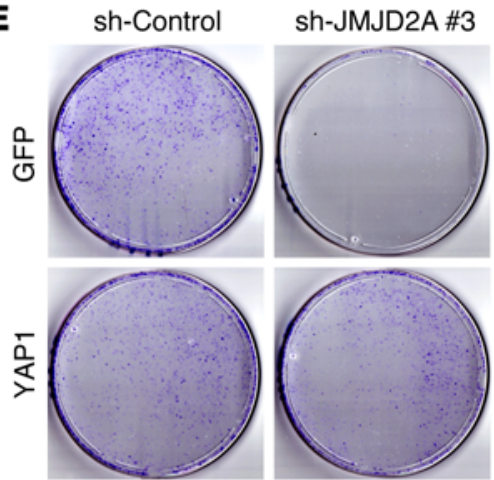

$\mathbf{F}$
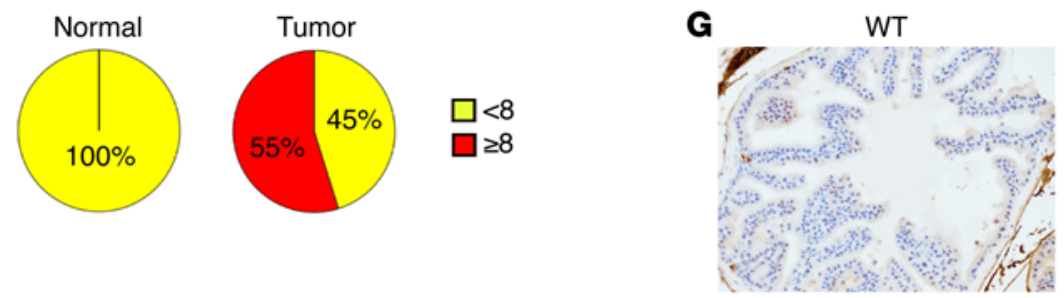

JMJD2A transgenic

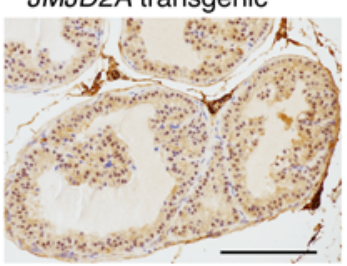

Figure 7. Role of YAP1 in prostate cancer. (A) Downregulation of YAP1 reduces and (B) overexpression of YAP1 stimulates LNCaP cell growth. Representative Western blots of shRNA-mediated knockdown of YAP1 (A) and HA-YAP1 expression (B) are shown. ${ }^{*} P<0.0001$, 1-way ANOVA (A); Student's $t$ test (B). Shown are averages ( $n=3$ per group) with SD. Representative of 3 independent experiments. (C) LNCaP cell growth after addition of $0-24 \mu M$ verteporfin dissolved in DMSO (20 mM stock). \#P=0.0002; ${ }^{*} P<0.0001$ (1-way ANOVA). Shown are averages ( $n=4$ per group) with SD. Representative of 2 independent experiments. (D) Ectopic YAP1, but not GFP, counteracts LNCaP growth inhibition caused by JMJD2A shRNA. Shown are averages ( $n=3$ per group) with SD. 1-way ANOVA. Representative of 2 independent experiments. (E) Likewise, YAP1 rescues LNCaP clonogenic capacity upon JMJD2A downregulation (representative out of 3 experiments). (F) Nuclear YAP1 overexpression in 31 cancerous prostate versus matching normal tissues (staining index $<8$ and $\geq 8 ; P=7.2 \times 10^{-7}, 2$-tailed Fisher exact probability test). (C) Example of YAP1 immunohistochemical staining in prostates from a WT or JMJD2A transgenic mouse. Scale bar: $0.1 \mathrm{~mm}$.

increase in $\mathrm{H} 3 \mathrm{~K} 9$ and $\mathrm{H} 3 \mathrm{~K} 36$ trimethylation, whereas $\mathrm{H} 3 \mathrm{~K} 4 \mathrm{me}_{3}$ levels were unaffected. This supports the notion that JMJD2A demethylates H3K9/K36 at the YAP1 promoter and thereby modulates its epigenetic status. On the other hand, downregulation of ETV1 not only reduced binding of ETV1 to the YAP1 promoter, but also of JMJD2A (Figure 6D and Supplemental Figure 21), corroborating that ETV1 is largely responsible for recruitment of JMJD2A to the YAP1 promoter in vivo; accordingly, H3K9 and H3K36 trimethylation were also enhanced in the presence of ETV1 shRNAs. Together, these data provide evidence that the YAP1 gene promoter can be epigenetically regulated by JMJD2A upon its recruitment by ETV1.
Role of YAP1 in prostate cancer. To define the role of YAP1 in prostate cancer cells, we expressed respective shRNAs and observed a reduction of LNCaP cell growth (Figure 7A). Conversely, YAP1 overexpression stimulated LNCaP cell growth (Figure 7B), which is consistent with a recently published report (35). Further, verteporfin, a small molecule that inhibits YAP1 function by compromising its interaction with TEAD transcription factors (36), phenocopied YAP1 downregulation in LNCaP cells (Figure 7C). Together, these data indicate that YAP1 is a progrowth molecule in prostate cancer cells and that its downregulation may be crucial for the growth-suppressive effects observed with JMJD2A shRNAs. 


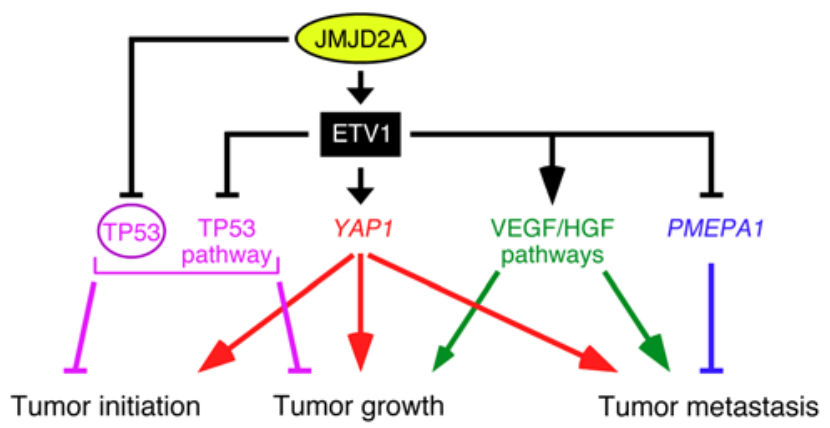

Figure 8. Model. Pleiotropic mechanisms by which JMJD2A promotes tumorigenesis.

To test this, we coexpressed JMJD2A shRNA together with a YAP1 expression vector in LNCaP cells. And indeed, YAP1 overexpression rescued the growth-repressive effect of JMJD2A shRNA to a large extent: growth repression on day 7 was reduced from $67 \%$ to $35 \%$ (Figure 7D). Similarly, YAP1 overexpression effectively counteracted the reduction of clonogenic capacity caused by JMJD2A shRNA (Figure 7E). These data strongly implicate YAP1 as an important downstream effector of JMJD2A.

Immunohistochemical analysis revealed that YAP1 was significantly overexpressed in human prostate tumors compared with matching normal tissue (Figure $7 \mathrm{~F}$ and Supplemental Figure 22A). Of note, JMJD2A protein expression positively correlated with that of YAP1 (Supplemental Figure 22B), consistent with JMJD2A influencing YAP1 transcription in the human prostate. Similarly, YAP1 was also overexpressed in JMJD2A transgenic mice (Figure $7 G$ ), further arguing for a causal relationship between JMJD2A overexpression and YAP1 upregulation. Moreover, high YAP1 expression correlated with disease recurrence after radical prostatectomy and also with Gleason score (Supplemental Figure 22, C and D). These correlations imply that YAP1 promotes the aggressiveness of prostate tumors. Like YAP1 protein expression, PMEPA1 mRNA levels were upregulated in prostate carcinomas compared with normal prostate tissue (Supplemental Figure 23A). However, a significant reduction of PMEPA1 expression was observable upon metastasis (Supplemental Figure 23, B and C), suggesting that JMJD2A- and ETV1-mediated downregulation of PMEPA1 could be important for the progression of localized prostate cancer to the advanced stage.

\section{Discussion}

Here, we uncovered several important new insights into prostate cancer pathophysiology and the molecular function of JMJD2A. First, JMJD2A overexpression induces prostatic neoplasia in a transgenic mouse model, establishing JMJD2A as an initiator of prostate tumorigenesis. Second, overexpression of JMJD2A occurs in the majority of human prostate tumors and correlates with Gleason score and metastasis, suggesting that JMJD2A might be a marker for and contributes to the progression to advanced prostate cancer. Third, JMJD2A is a novel ETV1binding protein and modulates ETV1 transcriptional responses. Fourth, the JMJD2A-ETV1 complex drives prostate carcinoma formation upon Pten haploinsufficiency. Fifth, YAP1 is a seminal downstream effector of JMJD2A, thus linking JMJD2A and ETV1 to the Hippo signaling pathway.

JMJD2A in prostate cancer. Recruitment of JMJD2A to chromatin can potentially lead to the demethylation of trimethylated histone $\mathrm{H} 3$ lysines 9 and $36(15,16)$. Consistently, we found that $\mathrm{H} 3 \mathrm{~K} 9 \mathrm{me}_{3}$ and $\mathrm{H} 3 \mathrm{~K} 36 \mathrm{me}_{3}$ were enhanced at the YAP1 promoter upon JMJD2A downregulation (Figure 6D), whereas global levels of these epigenetic marks were unaffected (Figure $3 \mathrm{~A}$ ). $\mathrm{H} 3 \mathrm{~K} 9 \mathrm{me}_{3}$ is generally associated with a repressed chromatin status, and $\mathrm{H} 3 \mathrm{~K} 36 \mathrm{me}_{3}$ may also inhibit transcription at the start site (11). Hence their demethylation by JMJD2A could promote transcription of target genes such as YAP1. On the other hand, JMJD2A can also suppress gene expression, as in the case of PMEPA1. This may involve demethylation of $\mathrm{H} 3 \mathrm{~K} 36 \mathrm{me}_{3}$ within the gene body, leading to reduced transcription elongation (37), or the recruitment of nuclear receptor corepressors and deacetylases that remove activating posttranslational modifications from histones $(38,39)$. This predicts that JMJD2A overexpression changes the epigenetic status of prostate cells and thereby contributes to their neoplastic transformation. This may not be the only mechanism of JMJD2A's action in cancer cells, since JMJD2A can also impair DNA damage repair, lead to site-specific gene copy number increases, or enhance protein synthesis (40-42); more studies are needed to decipher the contribution of each of these mechanisms to the oncogenic potential of JMJD2A.

Our study has shown that JMJD2A physically and functionally interacts with the DNA-binding transcription factor ETV1. Further, downregulation of ETV1 led to reduced JMJD2A binding to the YAP1 promoter in vivo, indicating a crucial role of ETV1 in the recruitment of JMJD2A to chromatin. However, JMJD2A is unlikely to exclusively interact with ETV1 and thereby influence prostate cancer cell physiology and, accordingly, different DNAbinding proteins may likewise utilize JMJD2A as a transcriptional cofactor. One attractive alternative interaction partner may be the androgen receptor, which was previously identified as forming complexes with JMJD2A (43). Furthermore, ETV1 can bind to the androgen receptor and thereby affect androgen-dependent gene transcription $(6,7,44)$, suggesting that even a tripartite complex consisting of JMJD2A, ETV1, and androgen receptor may be involved in prostate tumorigenesis.

Regardless, JMJD2A transgenic mice developed high-grade PIN, demonstrating that JMJD2A overexpression is an underlying cause for the initiation of prostate tumorigenesis. This is the first demonstration, to our knowledge, that overexpression of any histone demethylase induces a neoplastic phenotype at the organismal level. Similarly to ETV1 transgenic mice that exclusively develop PIN $(6,20)$, no carcinoma formation was observable in $J M J D 2 A$ transgenic mice. This is not unexpected, since the development of prostate and other cancers requires several genetic changes to occur, although the number may be as low as 3 in solid tumors $(2,45)$. When we mimicked 3 genetic alterations with our JMJD2A/ETV1/Pten ${ }^{+/-}$mice, carcinoma formation was observable in approximately half of these animals, whereas combining only 2 of these genetic changes did not result in any significant prostate tumor formation. This indicates that JMJD2A not only contributes to the formation of PINs, but also to their progression to carcinomas in cooperation with other genetic mutations, underscoring 
the oncogenic character of JMJD2A. Furthermore, our mouse data reinforce that the complex formation between JMJD2A and ETV1 is biologically relevant in tumorigenesis. Based on the facts that JMJD2B and JMJD2C are functionally and structurally very similar to JMJD2A (13), that JMJD2B and JMJD2C are also upregulated in human prostate tumors, and that they stimulate ETV1's transcriptional activity as shown for the MMP1 and YAP1 promoters, one may hypothesize that JMJD2B and JMJD2C might be interchangeable with JMJD2A in prostate tumorigenesis.

Metastatic prostate cancer, although initially contained by androgen ablation therapy, is a highly lethal disease without other effective therapeutic options. Our data strongly suggest that JMJD2A is overexpressed in metastatic prostate tumors and inhibition of JMJD2A may therefore be a valid therapeutic approach. Notably, JMJD2A is an enzyme and thus amenable to inhibition by small molecules. Several such inhibitors of JMJD2A were identified in vitro, but have not yet been developed into therapeutics (13, 46-48). Improvements in specificity, bioavailability, and safety should occur before commencement of (pre-)clinical tests with JMJD2A inhibitors, and possibly our JMJD2A transgenic mice may prove valuable as a tool for assessing their efficacy.

Roles of JMJD2A target genes in cancer. YAP1 is a transcriptional cofactor, and its overexpression has been observed in several cancers (27). A previous study suggested that YAP1 expression is elevated in prostate tumors (49); this was corroborated by our immunohistochemistry results, and we additionally found an association of YAP1 expression with tumor aggressiveness. Further, we demonstrated that YAP1 downregulation reduced LNCaP cell growth, whereas YAP1 overexpression accelerated it, thereby establishing YAP1 as a progrowth factor in prostate cancer cells. Finally, YAP1 was capable of largely rescuing the antigrowth effect of JMJD2A downregulation in LNCaP cells, emphasizing that YAP1 is a pivotal downstream effector of JMJD2A. This notion is strongly supported by a recently published study indicating that YAP1 overexpression in the mouse prostate also leads to the formation of neoplasias (50). Thus, by upregulating YAP1 transcription, JMJD2A may stimulate tumor initiation, growth, and metastasis (Figure 8), since all of these processes are regulated by YAP1 (51). Together, these findings suggest that YAP1 is an attractive prostate cancer drug target in its own right. Notably, proof of principle has been provided that YAP1 is druggable: small molecules or peptides were found that prevented YAP1 from coactivating TEAD transcription factors and thereby suppressed YAP1-induced hepatomegaly or gastric tumor growth in vitro and in vivo $(36,52)$. The fact that the YAP1 inhibitor verteporfin can inhibit LNCaP (Figure 7C) and VCaP prostate cancer cell growth (50) further stresses that YAP1 inhibition could indeed be a valid new strategy for combatting prostate cancer. Interestingly, $\mathrm{VCaP}$ cells are characterized by overexpression of ERG (53), and this ETS transcription factor, like ETV1, is capable of stimulating YAP1 gene transcription (50). Hence, it is tempting to speculate that JMJD2A may not only exert its oncogenic role through ETV1, but also via ERG, the 2 ETS proteins most frequently overexpressed in prostate cancer (3).

In contrast with YAP1, PMEPA1 downregulation or overexpression did not affect LNCaP prostate cancer cell growth, suggesting that PMEPA1 does not influence tumor initiation and localized growth. Also, in contrast with YAP1, PMEPA1 transcription was repressed by the JMJD2A-ETV1 complex. This does not dovetail with our bioinformatical analyses showing PMEPA1 upregulation in prostate carcinomas compared with normal tissue, suggesting that PMEPA1 transcription is not governed by JMJD2A/ETV1 in primary prostate tumors. However, we and others (54) also demonstrate that PMEPA1 becomes downregulated in metastases, suggesting that PMEPA1 may block the progression from indolent to advanced prostate cancer. Consistently, PMEPA1 knockdown promotes the metastatic potential of PC3 prostate cancer cells, possibly by relieving repression of prometastatic, TGF- $\beta$-stimulated genes, and low PMEPA1 levels correlate with an adverse prognosis $(54,55)$. Hence, by repressing PMEPA1 transcription during later stages of the disease, JMJD2A/ETV1 may foster metastasis and thereby contribute to advanced prostate cancer (Figure 8).

In addition to YAP1 transcription, our microarray data imply that JMJD2A (and ETV1) can stimulate angiogenic pathways controlled by VEGF and HGF. Both VEGF and the receptor for HGF are known to be overexpressed, especially in prostate cancer patients with bone metastasis $(56,57)$, and attempts are underway to suppress their function in tumor growth and metastasis (25). This provides another potential mechanism for how the JMJD2A-ETV1 complex stimulates tumorigenesis (Figure 8). Our microarray data also revealed that JMJD2A overexpression leads to suppression of TP53-modulated pathways. This may be particularly important during the initiation and progression of tumor formation (26). Although further studies are needed to explore how JMJD2A affects TP53-modulated pathways, one mechanism could be binding of JMJD2A to the TP53 tumor suppressor that reportedly inhibits its activity (58).

Perspective. Our survey of 3,949 different mRNA microarrays revealed a strong correlation between ETV1 and YAP1 as well as between JMJD2A and YAP1 expression, emphasizing that the molecular mechanisms by which JMJD2A and ETV1 regulate YAP1 transcription that were identified in this study are not restricted to the prostate, but apply to many other organs. Thus, JMJD2A and ETV1 are in general implicated as modulators of the Hippo pathway and therefore potentially affect development, organ growth, and tissue homeostasis, which are all tightly controlled by the Hippo signaling pathway (28). Finally, aside from prostate cancer, JMJD2A was found overexpressed in other tumors, including breast and lung cancer $(59,60)$. Our study predicts that JMJD2A overexpression is also an underlying cause for tumor formation in these cases and involves dysregulation of the JMJD2A/ETV1/ YAP1 axis. Hence, inhibition of this axis may be clinically beneficial beyond prostate cancer.

\section{Methods}

Coimmunoprecipitation. 293T cells (obtained from ATCC) were grown in 6-cm dishes and transiently transfected by the calcium phosphate coprecipitation method (61) with $2 \mu \mathrm{g}$ Flag $-E T V 1$ and $4.5 \mu \mathrm{g}$ 6MycJMJD expression vectors along with $2.5 \mu \mathrm{g}$ pBluescript $\mathrm{KS}^{+}$(Stratagene). Immunoprecipitations were performed as described (59) using 9E10 Myc Ab (Sigma-Aldrich, M4439) and coprecipitated protein revealed by Western blotting using M2 Flag Ab (Sigma-Aldrich, F1804) and enhanced chemiluminescence (62). ETV1 Ab H-70 (Santa Cruz Biotechnology Inc., sc-28681) was employed for endogenous coimmunoprecipitations followed by Western blotting with JMJD2A Ab (Bethyl Laboratories, A300-861A). 
Recombinant protein production and GST pull-down assays. JMJD2A cDNA was cloned into Flag-His-Bac vector (a derivative of pFast$\mathrm{Bac}^{\mathrm{TM}} 1$; a gift from Michael Hamann, Department of Immunology, Mayo Clinic), thereby fusing a Flag-tag plus a 6His-tag onto the N terminus of JMJD2A. Recombinant baculovirus was then generated with the Bac-to-Bac System (Invitrogen). Infected Sf9 cells were grown in spinner culture for 48 to 96 hours at $27^{\circ} \mathrm{C}$ and His-tagged protein purified using $\mathrm{Ni}^{2+}$-NTA agarose (QIAGEN) according to standard procedures. ETV1 fused to GST was expressed in E. coli and purified utilizing glutathione agarose (Sigma-Aldrich. G4510) according to recommended procedures, dialyzed against $20 \mathrm{mM}$ HEPES ( $\mathrm{pH}$ 7.4), $50 \mathrm{mM} \mathrm{NaCl}, 10 \%$ glycerol, $1 \mathrm{mM} \mathrm{DTT}$, and $0.2 \mathrm{mM} \mathrm{PMSF}$, and stored at $-80^{\circ} \mathrm{C}(63)$. GST pull-down assays were then performed by first binding GST fusion proteins to glutathione agarose and then challenging these loaded beads with recombinant JMJD2A protein. After washing away unbound JMJD2A, protein complexes were boiled off with Laemmli sample buffer and subjected to SDS polyacrylamide gel electrophoresis followed by anti-Flag Western blotting (64).

Luciferase assays. BPH-1 cells (obtained from ATCC) were grown in 12 -well plates to approximately $30 \%$ confluency and then transfected utilizing $2 \mu \mathrm{g}$ polyethylenimine (MW 25,000; catalog 23966, Polysciences Inc.) with $250 \mathrm{ng}$ luciferase reporter construct, $0.75 \mu \mathrm{g}$ pBluescript $\mathrm{KS}^{+}, 25$ ng empty vector pEV3S or ETV1 expression plasmid, and 10 ng pEV3S or Flag-tagged JMJD2 expression constructs. LNCaP cells (obtained from ATCC) were grown in poly- $L$-lysinecoated 6-well plates to approximately $40 \%$ confluency and then transfected utilizing $8 \mu \mathrm{g}$ polyethylenimine with $500 \mathrm{ng}$ luciferase reporter construct, $1.5 \mu \mathrm{g}$ pBluescript $\mathrm{KS}^{+}$, and $2.5 \mathrm{ng}$ of empty vector pEV3S or ETV1 expression plasmid. Alternatively, LNCaP cells were similarly transfected in 12-well plates using half the amount of reagents, but with 5 ng pEV3S (65) or ETV1 expression plasmid plus 40 ng of pEV3S or Flag-tagged JMJD2 expression construct. At 36 hours after transfection, cells were lysed and luciferase activities determined as described (66).

Oncomine analysis. The Oncomine database (www.oncomine.org) was accessed and searched for expression levels with a $P$ value of less than 0.05. Thresholds for fold-change and gene rank as well as data type were set to "all." Statistical significance was provided by Oncomine in the form of a Student's $t$ test. For a more extensive description of Oncomine, the reader is referred to a published report (67).

Immunohistochemistry. The consecutively cut prostate tissue microarrays used (AccuMax A302IV; ISU Abxis) encompassed 32 cores in duplicate from human prostate tumors and 32 matching normal prostate tissue cores; since 1 normal tissue core was essentially without cells, only 31 pairs of tumor/normal tissue cores were included in all analyses. Tissue microarray no. 141 was used for YAP1 and no. 142 for JMJD2A staining. All staining was performed with a Leica BOND-III machine. An initial 40 minutes (JMJD2A) or 20 minutes (YAP1) treatment with Bond Epitope Retrieval Solution 1 (Leica Biosystems) was employed to stain human prostate tissue microarrays. Mouse monoclonal JMJD2A Abs were from Abcam (ab104831; 1:400), and rabbit polyclonal YAP1 Abs were from Santa Cruz Biotechnology Inc. (H-125, sc-15407; 1:100). Intensity was scored on a scale of 0 to 3 , and percentage of positive cells on a scale of 1 to 4 . The staining index was derived as the product of these individual scores. The staining index of the tumor was defined as the average of the 2 respective tumor tissue cores.
For staining of mouse tissue, 40 minutes (YAP1) or 20 minutes (Ki67, smooth muscle actin, JMJD2A) treatment with Bond Epitope Retrieval Solution 1 was utilized. The following Abs were employed: YAP1 rabbit polyclonal H-125 Ab (Santa Cruz Biotechnology Inc., sc-15407; 1:100); Ki67 rabbit monoclonal SP6 Ab (Abcam, ab16667; 1:50); smooth muscle actin mouse monoclonal 1A4 Ab (from Dako, M0851; 1:400); and JMJD2A rabbit polyclonal Ab (Bethyl, IHC-00188; 1:50).

Knockdown and overexpression experiments. All shRNAs were cloned into pSIREN-RetroQ (Clontech). The human sequences targeted by shRNAs were as follows: JMJD2A no. 2 (GATAGCCAATAGCGATAAG); JMJD2A no. 3 (GTTGAGGATGGTCTTACCT); JMJD2A no. 5 (GGACTTAGCTTCATAACTA); YAP1 no. 1 (CCACCAAGCTAGATAAAGA); YAP1 no. 2 (GCTTATAAGGCATGAGACA); and YAP1 no. 3 (AGTAATAGTTGGTTGTGAA). ETV1 shRNAs were as described (68). Retrovirus expressing these shRNAs was produced as described (69). Cells were infected twice and selected with $1 \mu \mathrm{g} / \mathrm{ml}$ puromycin. Cell growth was measured with the PrestoBlue Cell Viability Kit (Invitrogen) according to the manufacturer's instructions. Briefly, cells were seeded in 96-well plates (3,000 cells per well) and grown for indicated times; then PrestoBlue reagent was added and cells incubated for another 1 hour at $37^{\circ} \mathrm{C}$. Fluorescence was measured at $590 \mathrm{~nm}$ (excitation at $530 \mathrm{~nm}$ ). For clonogenicity assays, 20,000 cells were plated in a $10-\mathrm{cm}$ dish and the medium changed every 3 to 4 days. After 3 weeks, cells were fixed with $10 \%$ methanol $10 \%$ glacial acetic acid for 10 minutes and stained with $0.4 \%$ crystal violet in $10 \%$ ethanol for 5 minutes. In the case of overexpression experiments, cells were infected with lentivirus expressing either HA-tagged YAP1 or a GFP control.

Flow cytometry. Cell-cycle analysis was performed by measuring uptake of EdU (Click-iT EdU Alexa Fluor 647 flow cytometry kit, Invitrogen) and staining DNA with propidium iodide (PI). Cells were incubated with $20 \mu \mathrm{M}$ EdU for 1 hour at $37^{\circ} \mathrm{C}$ and harvested by trypsinization. Then cells were fixed in $4 \%$ paraformaldehyde for 15 minutes and permeabilized with saponin-based buffer; fluorescent labeling was performed by addition of Click-iT reaction mixtures. For DNA staining, cells were incubated in PI solution with RNase A for 30 minutes at $37^{\circ} \mathrm{C}$. Cells were sorted with a FACSCalibur (BD Biosciences) and cellcycle profiles determined with FlowJo analysis software (Tree Star).

Transgenic mice. Human JMJD2A cDNA was cloned downstream of the androgen-responsive and prostate-specific ARR2PB promoter (21) and upstream of a SV40 polyadenylation site and intron. This gene cassette was injected into 1-cell fertilized FVB mouse embryos that were reimplanted into pseudopregnant females. Resulting founder animals (FO) were crossed with C57BL/6 mice to generate F1 mice, which were backcrossed one more time with C57BL/6 mice, thereby obtaining F2 mice. These F2 mice were crossed among themselves to obtain F3 mice. F2 and F3 mice, which were all 25\% FVB and 75\% C57BL/6 in genetic background, were employed for analysis. For genotyping, clipped mouse tails ( $0.5 \mathrm{~cm}$ length) were boiled in 0.5 $\mathrm{ml}$ of $50 \mathrm{mM} \mathrm{NaOH}$ for 30 minutes, after which $0.1 \mathrm{ml}$ of $1 \mathrm{M} \mathrm{Tris-HCl}$ ( $\mathrm{pH} 8$ ) was added for neutralization. One microliter of supernatant was subjected to PCR amplification using the following primers: ARR2PBfor-1 (5'-GGAAGCTACTCTGCACCTTGTCAG-3') and JMJD2A-Kpn (5'-TCATAGAGGGTACCATTCACATCTGC- ${ }^{\prime}$ ), which resulted in a 508-bp PCR product identifying the presence of the JMJD2A transgene. PCR was performed with the GoTaq DNA polymerase kit (Pro- 
mega M3008) utilizing the following temperature program: $95^{\circ} \mathrm{C}$ for 2 minutes; 10 cycles of $94^{\circ} \mathrm{C}$ for 40 seconds, $68^{\circ} \mathrm{C}\left(-1^{\circ} \mathrm{C}\right.$ per cycle $)$ for 30 seconds, $72^{\circ} \mathrm{C}$ for 40 seconds; 30 cycles of $94^{\circ} \mathrm{C}$ for 40 seconds, $58^{\circ} \mathrm{C}$ for 30 seconds, $72^{\circ} \mathrm{C}$ for 40 seconds ( +1 s per cycle); $72^{\circ} \mathrm{C}$ for 4 minutes, followed by cooling down to $4^{\circ} \mathrm{C}$. In addition, JMJD2A transgenic mice (502 line) were crossed with ETV1 transgenic mice (1919 line; described in ref. 6) and Pten $^{+/-}$heterozygous knockouts to establish compound mice, which were then utilized for analysis at around 1 year of age. Pten $^{+/-}$mice (70) were obtained from the NCI Mouse Repository (strain number 01XH3) and backcrossed onto C57BL/6 mice for at least 3 generations before utilization in our experiments. Genotyping for the ETV1 transgene was done with ARR2PB-for-1 and hETV1-661-rev (5'-GGATGTTTGGCTCAGACATCTGG-3') primers, yielding a 757-bp PCR product, and Pten status was assessed with a 3-primer combination (Pten-1, 5'-TTGCACAGTATCCTTTTGAAG-3'; Pten-2, 5'-GTCTCTGGTCCTTACTTCC-3'; Pten-3, 5'-ACGAGACTAGTGAGACGTGC-3'), with an approximately 200/400-bp PCR product indicating the WT/knockout Pten allele.

Electrophoretic mobility shift assay for DNA binding. Oligonucleotide "up" and "down" pairs were hybridized to generate doublestranded oligonucleotides and labeled with ${ }^{32} \mathrm{P}$-dATP utilizing Klenow DNA polymerase. The corresponding sequences were as follows (ETS core sequences in bold; mutated nucleotides underlined): E1/2-up (5'-AGCGGAGCGGAAGAACTTCCTGCAGCCA-3') and E1/2-down (5'-CTTGGCTGCAGGAAGTTCTTCCGCTCCGCT-3'); Em1/2-up (5'-AGCGGAGCGGTAGAACTTCCTGCAGCCA-3') and Em1/2-down (5'-CTTGGCTGCAGGAAGTTCTACCGCTCCGCT3'); E1/m2-up (5'-AGCGGAGCGGAAGAACTACCTGCAGCCA-3') and E1/m2-down (5'-CTTGGCTGCAGGTAGTTCTTCCGCTCCGCT-3');E3-up(5'-GTTCGGACCCGGATTGGACCC-3') andE3-down (5'-GATGGGTCCAATCCGGGTCCGA-3'); E4-up (5'-AGTGTGCAGGAATGTAGCA-3') and E4-down (5'-AGTTGCTACATTCCTGCAC-3'); E5-up (5'-CTTGCAGCGAAAAGTTTCCCTGCGCTG-3') and E5-down (5'-CAGCGCAGGGAAACTTTTCGCTGCA-3'); E6/7up (5'-GCGCAGAGGAAGGAAGAGCCGAG-3') and E6/7-down (5'-CTCTCGGCTCTTCCTTCCTCTGCGC-3'); Em6/7-up (5'GCGCGACGAAGGAAGAGCCGAG-3') and Em6/7-down (5'CTCTCGGCTCTTCCTTCGTCTGCGC-3'); E6/m7-up (5'-GCGCAGAGGAAGGACGAGCCGAG-3') and E6/m7-down (5'-CTCTCGGCTCGTCCTTCCTCTGCGC-3'); E8-up (5'-GCCGCCAGGGAAAAGAA-3') and E8-down (5'-CTTTCTTTTCCCTGGCGGC3'). The E74 "up" and "down" oligonucleotide sequences have been described (14). Binding reactions were conducted in $10 \mu \mathrm{l}$ of $20 \mathrm{mM}$ HEPES (pH 7.4), 25 mM NaCl, 0.5 mM EDTA, 12\% glycerol, $0.01 \%$ Tween 20, $0.1 \mu \mathrm{g} / \mu \mathrm{l} \mathrm{BSA}, 0.05 \mu \mathrm{g} / \mu \mathrm{l}$ poly $(\mathrm{dI}-\mathrm{dC}) \cdot \operatorname{poly}(\mathrm{dI}-\mathrm{dC})$, and 2 mM DTT containing approximately $0.25 \mathrm{ng}$ 32 P-labeled double-stranded oligonucleotide. Bacterially expressed and purified ETV1 encompassing amino acids 249-477 and $0.4 \mu \mathrm{l}$ of anti-ETV1 Ab (C-20; sc-1953, Santa Cruz Biotechnology Inc.) were employed as indicated. After 30 minutes on ice, reaction mixes were applied onto $3.5 \%$ to $4 \%$ acrylamide gels (acrylamide/bisacrylamide $=37.5: 1$ ) in $0.5 \times \mathrm{TBE}$ and electrophoresed at $4^{\circ} \mathrm{C}$. Gels were dried and then exposed to film at $-80^{\circ} \mathrm{C}(71)$.

Gene expression analyses. Microarray experiments were conducted with Illumina Human BeadChip (HT-12 v4; 47,000 probes); all original microarray data were deposited in the NCBI's Gene Expression Omnibus (GEO GSE47750). Analysis of global gene-gene correlation levels was conducted as described previously (32). Briefly, all curated $\left({ }^{*}\right.$.GDS files) human 2 -color data sets were downloaded from GEO. After quality checking, 3,949 individual microarray experiments remained, which were then normalized and analyzed for Pearson correlations.

Total RNA for RT-PCR experiments was isolated with TRIzol (Life Technologies) from LNCaP cells (72), and approximately $1 \mu \mathrm{g}$ RNA was reverse transcribed using GoScript reverse transcriptase (Promega, A5004) and $\mathrm{pd}(\mathrm{N})_{6}$ random primers (Roche Applied Science, 11034731001) following the manufacturer's instructions. Subsequent PCR was performed using the GoTaq DNA polymerase kit and the following temperature program: $95^{\circ} \mathrm{C}$ for 3 minutes; indicated number of cycles of $94^{\circ} \mathrm{C}$ for 30 seconds, $57^{\circ} \mathrm{C}$ for 30 seconds, $72^{\circ} \mathrm{C}$ for $30 \mathrm{sec}-$ onds; $72^{\circ} \mathrm{C}$ for 4 minutes, followed by cooling down to $4^{\circ} \mathrm{C}$. Forward and reverse primers employed were as follows: YAP1 (5'-GAACTCGGCTTCAGGTCCTC-3' and 5'-GTTGCTGCTGGTTGGAGTTG-3'; 25 cycles; 239-bp product); PMEPA1 (5'-GACAGTCTCCTGCGAAACCA-3' and 5'-CCACAGGCATCCTTCTGAGG-3'; 35 cycles; 198-bp product); JMJD2A (5'-CGAGAGTTCCGCAAGATAGC-3' and 5'-CTCCTTTTCCACCAAGTCCA-3'; 30 cycles; 207-bp product); ETV1 (5'-CCAGCTTTCTGAACCCTGTAACTC-3' and 5'-CATATGCAAAATCTCTGGGTTCCTG-3'; 25 cycles; 233-bp product); MMP7 (5'-TGTGGAGTGCCAGATGTTGCAG-3' and 5'-CTAAATGGAGTGGAGGAACAGTGC-3'; 40 cycles; 642-bp product); and $G A P D H$ (5'-GAGCCACATCGCTCAGACACC-3' and 5'-TGACAAGCTTCCCGTTCTCAGC-3'; 20 cycles; 226-bp product).

ChIP assay. LNCaP cells were treated with formaldehyde and then processed for ChIP essentially as described (73). To amplify immunoprecipitated promoter DNA fragments, nested PCR reactions were performed. The PCR program was as follows: $98^{\circ} \mathrm{C}$ for 2 minutes; 8 cycles of $98^{\circ} \mathrm{C}$ for 30 seconds, $65^{\circ} \mathrm{C}\left(-1^{\circ} \mathrm{C}\right.$ per cycle $)$ for 30 seconds, $72^{\circ} \mathrm{C}$ for 30 seconds; 20 cycles (for the first PCR) or 25-35 cycles (for the nested PCR) of $98^{\circ} \mathrm{C}$ for 30 seconds, $57^{\circ} \mathrm{C}$ for 30 seconds, $72^{\circ} \mathrm{C}$ for 25 seconds ( +1 second per cycle); and $72^{\circ} \mathrm{C}$ for 4 minutes, followed by cooling down to $4^{\circ} \mathrm{C}$. The reaction volume was $25 \mu$ l containing $250 \mathrm{ng}$ of each primer and either 0.5 to $5 \mu \mathrm{l}$ of ChIP-DNA or $0.5 \mu$ l product of the first PCR; iProof high-fidelity DNA polymerase (BioRad; 0.8 units) was employed for the first PCR, while the second PCR was performed with either iProof or GoTaq DNA polymerase. MMP7 ChIP primers have been described (31). For YAP1, the following primers were used: YAP1-2561-for (5'-GGCGAACTGGAAGCGCCTTTCC-3') and YAP12989-rev (5'-GAGACAGAAACTCGCCTCAAACGC-3'); followed by nested PCR with YAP1-2610-for (5'-GCGGAGCGGAAGAACTTCCTGC-3') and YAP1-2989-rev, resulting in a 380-bp product. In some cases, instead of YAP1-2989-rev, YAP1-2845-rev (5'-CGCTGCAAGTTGCTACATTCCTGC-3') was utilized in the nested PCR, resulting in a 236-bp product. For YAP1 upstream, the following primers were used: YAP1-242-for (5'-GCATGTTTTGAGATAGTGATCTACCTG-3') and YAP1-660-rev (5'-TAGAAGCTGTCCCTTTACTAGAGC-3'); followed by nested PCR with YAP1-333-for (5'-GATGCTGCCAACAGATAATTGGTCCAC-3') and YAP1-660-rev, resulting in a 328-bp product. For YAP1 intron 3, the following primers were used: YAP1-74618-for (5'-CAAAGTGCTGGGATTACAGGCGTGAG-3') and YAP1-74923rev (5'-CAAGGGGGGAAATGTAGGATTACCAC-3'), followed by nested PCR with YAP1-74678-for (5'-CAGTGGTTCCTTAGACCGGCATCAGC-3') and YAP1-74923-rev, resulting in a 246-bp product. For PMEPA1, the following primers were used: PMEPA1-2351-for (5'-CAGCTATTTACATGACAAGGGCTTC-3') and PMEPA1-2827-rev 
(5'-GGCTCTTAAAGGGCCAGAGCTAGG-3'), followed by nested PCR with PMEPA1-2464-for (5'-CCGAGCTGCTGGAGAACTGAAGG-3') and PMEPA1-2748-rev (5'-CCAACGGCGGTCAGCTTGGAACG-3'), resulting in a 285-bp product.

Statistics. Statistical evaluation was performed as indicated. A $P$ value of less than 0.05 was considered significant. All Student's $t$ tests were unpaired and 2 sided. ANOVA was 1-way and always used with Bonferroni's correction.

Study approval. Experiments in mice were carried out with the approval of the Institutional Animal Care and Use Committee at the University of Oklahoma Health Sciences Center.

\section{Author contributions}

TDK, FJ, SS, SO, JDW, and RJ designed and performed the experiments. TDK, FJ, SS, SO, SAL, JPG, AJJ, JMVD, JDW, and RJ analyzed the data. RJ wrote the paper with comments from all other authors and directed the project.

\section{Acknowledgments}

We thank Lawrence Rothblum and Leonidas Tsiokas for critical comments on this manuscript, Bart Frank for help with microarray experiments, Christopher Aston for statistical advice, and the Pathology Core at the Stephenson Cancer Center, which was supported by the National Institute of General Medical Sciences (P20 GM103639), for performing immunohistochemistry. This work was funded by grants from the Stephenson Cancer Center and the National Cancer Institute of the NIH (R01 CA154745 to $\mathrm{R}$. Janknecht). The content is solely the responsibility of the authors and does not necessarily represent the official views of the granting agencies.

Address correspondence to: Ralf Janknecht, University of Oklahoma Health Sciences Center, 975 NE 10th Street, Oklahoma City, Oklahoma 73104, USA. Phone: 405.271.2377; E-mail: ralf-janknecht@ouhsc.edu.
1. Ryan CJ, Tindall DJ. Androgen receptor rediscovered: the new biology and targeting the androgen receptor therapeutically. JClin Oncol. 2011;29(27):3651-3658.

2. Shen MM, Abate-Shen C. Molecular genetics of prostate cancer: new prospects for old challenges. Genes Dev. 2010;24(18):1967-2000.

3. Clark JP, Cooper CS. ETS gene fusions in prostate cancer. Nat Rev Urol. 2009;6(8):429-439.

4. Hollenhorst PC, McIntosh LP, Graves BJ. Genomic and biochemical insights into the specificity of ETS transcription factors. Annu Rev Biochem. 2011;80:437-471.

5. Oh S, Shin S, Janknecht R. ETV1, 4 and 5: An oncogenic subfamily of ETS transcription factors. Biochim Biophys Acta. 2012;1826(1):1-12.

6. Shin S, et al. Induction of prostatic intraepithelial neoplasia and modulation of androgen receptor by ETS variant $1 /$ ETS-related protein 81 . Cancer Res. 2009;69(20):8102-8110.

7. Baena E, et al. ETV1 directs androgen metabolism and confers aggressive prostate cancer in targeted mice and patients. Genes Dev. 2013;27(6):683-698.

8. Dawson MA, Kouzarides T. Cancer epigenetics: from mechanism to therapy. Cell. 2012; 150(1):12-27.

9. Seligson DB, et al. Global histone modification patterns predict risk of prostate cancer recurrence. Nature. 2005;435(7046):1262-1266.

10. Ellinger J, et al. Global levels of histone modifications predict prostate cancer recurrence. Prostate. 2010;70(1):61-69.

11. Chi P, Allis CD, Wang GG. Covalent histone modifications - miswritten, misinterpreted and mis-erased in human cancers. Nat Rev Cancer. 2010;10(7):457-469.

12. Kooistra SM, Helin K. Molecular mechanisms and potential functions of histone demethylases. Nat Rev Mol Cell Biol. 2012;13(5):297-311.

13. Berry WL, Janknecht R. KDM4/JMJD2 histone demethylases: epigenetic regulators in cancer cells. Cancer Res. 2013;73(10):2936-2942.

14. Bosc DG, Goueli BS, Janknecht R. HER2/Neumediated activation of the ETS transcription factor ER81 and its target gene MMP-1. Oncogene.
2001;20(43):6215-6224.

15. Whetstine JR, et al. Reversal of histone lysine trimethylation by the JMJD2 family of histone demethylases. Cell. 2006;125(3):467-481.

16. Klose RJ, et al. The transcriptional repressor JHDM3A demethylates trimethyl histone $\mathrm{H} 3$ lysine 9 and lysine 36. Nature. 2006;442(7100):312-316.

17. Cloos PA, et al. The putative oncogene GASC1 demethylates tri- and dimethylated lysine 9 on histone H3. Nature. 2006;442(7100):307-311.

18. Lapointe J, et al. Gene expression profiling identifies clinically relevant subtypes of prostate cancer. Proc Natl Acad Sci U S A. 2004;101(3):811-816.

19. Cai C, et al. ETV1 is a novel androgen receptorregulated gene that mediates prostate cancer cell invasion. Mol Endocrinol. 2007;21(8):1835-1846.

20. Tomlins SA, et al. Distinct classes of chromosomal rearrangements create oncogenic ETS gene fusions in prostate cancer. Nature. 2007;448(7153):595-599.

21. Zhang J, Thomas TZ, Kasper S, Matusik RJ. A small composite probasin promoter confers high levels of prostate-specific gene expression through regulation by androgens and glucocorticoids in vitro and in vivo. Endocrinology. 2000;141(12):4698-4710.

22. Park JH, et al. Prostatic intraepithelial neoplasia in genetically engineered mice. Am J Pathol. 2002;161(2):727-735.

23. Di Cristofano A, Pesce B, Cordon-Cardo C, Pandolfi PP. Pten is essential for embryonic development and tumour suppression. Nat Genet. 1998;19(4):348-355.

24. Trotman LC, et al. Pten dose dictates cancer progression in the prostate. PLoS Biol. 2003;1(3):E59.

25. Antonarakis ES, Carducci MA. Targeting angiogenesis for the treatment of prostate cancer. Expert Opin Ther Targets. 2012;16(4):365-376.

26. Vazquez A, Bond EE, Levine AJ, Bond GL. The genetics of the 553 pathway, apoptosis and cancer therapy. Nat Rev Drug Discov. 2008;7(12):979-987.

27. Harvey KF, Zhang X, Thomas DM. The Hippo pathway and human cancer. Nat Rev Cancer. 2013;13(4):246-257.

28. Yu FX, Guan KL. The Hippo pathway: regulators and regulations. Genes Dev. 2013;27(4):355-371.

29. Xu LL, et al. PMEPA1, an androgen-regulated NEDD4-binding protein, exhibits cell growth inhibitory function and decreased expression during prostate cancer progression. Cancer Res. 2003;63(15):4299-4304.

30. Li H, et al. A feedback loop between the androgen receptor and a NEDD4-binding protein, PMEPA1, in prostate cancer cells. J Biol Chem. 2008;283(43):28988-28995.

31. Shin S, Oh S, An S, Janknecht R. ETS variant 1 regulates matrix metalloproteinase-7 transcription in LNCaP prostate cancer cells. Oncol Rep. 2013;29(1):306-314.

32. Wren JD. A global meta-analysis of microarray expression data to predict unknown gene functions and estimate the literature-data divide. Bioinformatics. 2009;25(13):1694-1701.

33. Janknecht R. Analysis of the ERK-stimulated ETS transcription factor ER81. Mol Cell Biol. 1996;16(4):1550-1556.

34. Dowdy SC, Mariani A, Janknecht R. HER2/ Neu- and TAK1-mediated up-regulation of the transforming growth factor beta inhibitor Smad7 via the ETS protein ER81. J Biol Chem. 2003;278(45):44377-44384.

35. Zhang L, et al. The Hippo pathway effector YAP regulates motility, invasion, and castrationresistant growth of prostate cancer cells. Mol Cell Biol. 2015;35(8):1350-1362.

36. Liu-Chittenden Y, et al. Genetic and pharmacological disruption of the TEAD-YAP complex suppresses the oncogenic activity of YAP. Genes Dev. 2012;26(12):1300-1305.

37. Wagner EJ, Carpenter PB. Understanding the language of Lys36 methylation at histone H3. Nat Rev Mol Cell Biol. 2012;13(2):115-126.

38. Gray SG, et al. Functional characterization of JMJD2A, a histone deacetylase- and retinoblastoma-binding protein. J Biol Chem. 2005;280(31):28507-28518.

39. Zhang D, Yoon HG, Wong J. JMJD2A is a novel $\mathrm{N}$-CoR-interacting protein and is involved in repression of the human transcription factor achaete scute-like homologue 2 (ASCL2/Hash2). Mol Cell Biol. 2005;25(15):6404-6414. 
40. Mallette FA, et al. RNF8- and RNF168-dependent degradation of KDM4A/JMJD2A triggers $53 \mathrm{BP} 1$ recruitment to DNA damage sites. ЕМВО J. 2012;31(8):1865-1878.

41. Black JC, et al. KDM4A lysine demethylase induces site-specific copy gain and rereplication of regions amplified in tumors. Cell. 2013;154(3):541-555.

42. Van Rechem C, et al. Lysine demethylase KDM4A associates with translation machinery and regulates protein synthesis. Cancer Discov. 2015;5(3):255-263.

43. Shin S, Janknecht R. Activation of androgen receptor by histone demethylases JMJD2A and JMJD2D. Biochem Biophys Res Commun. 2007;359(3):742-746.

44. Chen Y, et al. ETS factors reprogram the androgen receptor cistrome and prime prostate tumorigenesis in response to PTEN loss. Nat Med. 2013;19(8):1023-1029.

45. Tomasetti C, Marchionni L, Nowak MA, Parmigiani G, Vogelstein B. Only three driver gene mutations are required for the development of lung and colorectal cancers. Proc Natl Acad Sci US A. 2015;112(1):118-123.

46. Suzuki T, Miyata N. Lysine demethylases inhibitors. J Med Chem. 2011;54(24):8236-8250.

47. Labbe RM, Holowatyj A, Yang ZQ. Histone lysine demethylase (KDM) subfamily 4 : structures, functions and therapeutic potential. Am J Transl Res. 2013;6(1):1-15.

48. Wang L, et al. A small molecule modulates Jumonji histone demethylase activity and selectively inhibits cancer growth. Nat Commun. 2013;4:2035.

49. Zhao B, et al. Inactivation of YAP oncoprotein by the Hippo pathway is involved in cell contact inhibition and tissue growth control. Genes Dev. 2007;21(21):2747-2761.

50. Nguyen LT, et al. ERG activates the YAP1 transcriptional program and induces the development of age-related prostate tumors. Cancer Cell. 2015;27(6):797-808.

51. Moroishi T, Hansen CG, Guan KL. The emerging roles of YAP and TAZ in cancer. Nat Rev Cancer. 2015;15(2):73-79.

52. Jiao $S$, et al. A peptide mimicking VGLL4 function acts as a YAP antagonist therapy against gastric cancer. Cancer Cell. 2014;25(2):166-180.

53. Tomlins SA, et al. Recurrent fusion of TMPRSS2 and ETS transcription factor genes in prostate cancer. Science. 2005;310(5748):644-648.

54. Fournier PGJ, et al. The TGF- $\beta$ signaling regulator PMEPA1 suppresses prostate cancer metastases to bone. Cancer Cell. 2015;27(6):809-821.

55. Watanabe $Y$, et al. TMEPAI, a transmembrane TGF- $\beta$-inducible protein, sequesters Smad proteins from active participation in TGF-beta signaling. Mol Cell. 2010;37(1):123-134.

56. Knudsen BS, et al. High expression of the Met receptor in prostate cancer metastasis to bone. Urology. 2002;60(6):1113-1117.

57. Tomic TT, Gustavsson H, Wang W, Jennbacken $\mathrm{K}$, Welen K, Damber JE. Castration resistant prostate cancer is associated with increased blood vessel stabilization and elevated levels of VEGF and Ang-2. Prostate. 2012;72(7):705-712.

58. Kim TD, Shin S, Berry WL, Oh S, Janknecht R. The JMJD2A demethylase regulates apoptosis and proliferation in colon cancer cells. J Cell Biochem. 2012;113(4):1368-1376.

59. Berry WL, Shin S, Lightfoot SA, Janknecht R. Oncogenic features of the JMJD2A histone demethylase in breast cancer. Int JOncol. 2012;41(5):1701-1706.

60. Mallette FA, Richard S. JMJD2A promotes cellular transformation by blocking cellular senescence through transcriptional repression of the tumor suppressor CHD5. Cell Rep. 2012;2(5):1233-1243.

61. Janknecht R. Regulation of the ER81 transcription factor and its coactivators by mitogen- and stress-activated protein kinase 1 (MSK1). Oncogene. 2003;22(5):746-755.

62. Papoutsopoulou S, Janknecht R. Phosphorylation of ETS transcription factor ER81 in a complex with its coactivators CREB-binding protein and p300. Mol Cell Biol. 2000;20(19):7300-7310.

63. Goel A, Janknecht R. Acetylation-mediated tran- scriptional activation of the ETS protein ER81 by p300, P/CAF, and HER2/Neu. Mol Cell Biol. 2003;23(17):6243-6254.

64. Berry WL, Kim TD, Janknecht R. Stimulation of beta-catenin and colon cancer cell growth by the KDM4B histone demethylase. Int JOncol. 2014;44(4):1341-1348.

65. Matthias P, Muller MM, Schreiber E, Rusconi S, Schaffner W. Eukaryotic expression vectors for the analysis of mutant proteins. Nucleic Acids Res. 1989;17(15):6418.

66. Wu J, Janknecht R. Regulation of the ETS transcription factor ER81 by the 90-kDa ribosomal S6 kinase 1 and protein kinase A. J Biol Chem. 2002;277(45):42669-42679.

67. Rhodes DR, et al. Oncomine 3.0: genes, pathways, and networks in a collection of 18,000 cancer gene expression profiles. Neoplasia. 2007;9(2):166-180.

68. Oh S, Shin S, Lightfoot SA, Janknecht R. 143-3 proteins modulate the ETS transcription factor ETV1 in prostate cancer. Cancer Res. 2013;73(16):5110-5119.

69. Kim TD, Oh S, Shin S, Janknecht R. Regulation of tumor suppressor p53 and HCT116 cell physiology by histone demethylase JMJD2D/KDM4D. PLoS One. 2012;7(4):e34618.

70. Podsypanina K, et al. Mutation of Pten/Mmac1 in mice causes neoplasia in multiple organ systems. Proc Natl Acad Sci U S A. 1999;96(4):1563-1568.

71. DiTacchio L, Bowles J, Shin S, Lim DS, Koopman P, Janknecht R. Transcription factors ER71/ETV2 and SOX9 participate in a positive feedback loop in fetal and adult mouse testis. J Biol Chem. 2012;287(28):23657-23666.

72. Goel A, Janknecht R. Concerted activation of ETS protein ER 81 by 160 coactivators, the acetyltransferase p300 and the receptor tyrosine kinase HER2/Neu. J Biol Chem. 2004;279(15):14909-14916.

73. Goueli BS, Janknecht R. Regulation of telomerase reverse transcriptase gene activity by upstream stimulatory factor. Oncogene. 2003;22(39):8042-8047. 